\title{
HYDROSTATIC GAS CONSTRAINTS ON SUPERMASSIVE BLACK HOLE MASSES: IMPLICATIONS FOR HYDROSTATIC EQUILIBRIUM AND DYNAMICAL MODELING IN A SAMPLE OF EARLY-TYPE GALAXIES
}

\author{
Philip J. Humphrey ${ }^{1}$, David A. Buote ${ }^{1}$, Fabrizio Brighenti ${ }^{2,3}$, Karl Gebhardt ${ }^{4}$, And William G. Mathews ${ }^{3}$ \\ ${ }^{1}$ Department of Physics and Astronomy, University of California at Irvine, 4129 Frederick Reines Hall, Irvine, CA 92697-4575, USA \\ 2 Dipartimento di Astronomia, Università di Bologna, Via Ranzani 1, Bologna 40127, Italy \\ ${ }^{3}$ University of California Observatories, Lick Observatory, University of California, Santa Cruz, CA 95064, USA \\ ${ }^{4}$ Astronomy Department, University of Texas, Austin, TX 78712, USA \\ Received 2009 June 2; accepted 2009 August 11; published 2009 September 8
}

\begin{abstract}
We present new mass measurements for the supermassive black holes (SMBHs) in the centers of three early-type galaxies. The gas pressure in the surrounding, hot interstellar medium (ISM) is measured through spatially resolved spectroscopy with the Chandra X-ray Observatory, allowing the SMBH mass $\left(M_{\mathrm{BH}}\right)$ to be inferred directly under the hydrostatic approximation. This technique does not require calibration against other SMBH measurement methods and its accuracy depends only on the ISM being close to hydrostatic, which is supported by the smooth X-ray isophotes of the galaxies. Combined with results from our recent study of the elliptical galaxy NGC 4649, this brings the number of galaxies with SMBHs measured in this way to four. Of these, three already have mass determinations from the kinematics of either the stars or a central gas disk, and hence join only a handful of galaxies with $M_{\mathrm{BH}}$ measured by more than one technique. We find good agreement between the different methods, providing support for the assumptions implicit in both the hydrostatic and the dynamical models. The stellar mass-to-light ratios for each galaxy inferred by our technique are in agreement with the predictions of stellar population synthesis models assuming a Kroupa initial mass function (IMF). This concurrence implies that no more than 10\%-20\% of the ISM pressure is nonthermal, unless there is a conspiracy between the shape of the IMF and nonthermal pressure. Finally, we compute Bondi accretion rates $\left(\dot{M}_{\text {bondi }}\right)$, finding that the two galaxies with the highest $\dot{M}_{\text {bondi }}$ exhibit little evidence of X-ray cavities, suggesting that the correlation with the active galactic nuclei jet power takes time to be established.
\end{abstract}

Key words: black hole physics - galaxies: elliptical and lenticular, cD - galaxies: individual (NGC1332, NGC4261, NGC4472, NGC4649) - galaxies: ISM - X-rays: galaxies

Online-only material: color figures

\section{INTRODUCTION}

Supermassive black holes (SMBHs) with masses ranging from less than a million to a few billion times the mass of the Sun, believed to be ubiquitous in galaxy bulges, are increasingly being recognized as essential ingredients in the formation of galaxies (e.g., Silk \& Rees 1998; Di Matteo et al. 2005; Hopkins et al. 2006). Compelling evidence for the close link between the formation of SMBHs and stellar spheroids is provided by the tight correlations observed between the SMBH mass, $M_{\mathrm{BH}}$, and the properties of the galaxy, such as the bulge luminosity, central light concentration, and, in particular, central velocity dispersion, $\sigma_{*}$ (Gebhardt et al. 2000a; Ferrarese \& Merritt 2000; Kormendy \& Richstone 1995; Marconi \& Hunt 2003; Graham et al. 2001). Since different physical processes involved in this co-evolution can lead to quantitatively different behavior, both the shape of these relations, particularly that between $M_{\mathrm{BH}}$ and $\sigma_{*}$, and the scatter about them have become crucial diagnostics for constraining our picture of galaxy formation (e.g., King 2003; Nipoti et al. 2003; Granato et al. 2004; Robertson et al. 2006). Unfortunately, controversies persist over the exact slope of the $M_{\mathrm{BH}}-\sigma_{*}$ relation (Merritt \& Ferrarese 2001; Tremaine et al. 2002; Ferrarese \& Ford 2005), and a number of authors have discussed possible deviations from the universal, loglinear form typically assumed (Wyithe 2006; Lauer et al. 2007; Hu 2008; Graham 2008). Furthermore, most authors neglect systematic uncertainties in the modeling techniques, and these could have an important effect (Gültekin et al. 2009).
Despite their central importance for understanding galaxy evolution, only $\sim 40-50$ precise, reliable SMBH measurements span $\sim 3$ orders of magnitude in $M_{\mathrm{BH}}$ (Ferrarese \& Ford 2005; Graham 2008; Gültekin et al. 2009). These are all derived from the kinematics of stars or a rotating central gas disk in the deep potential well near to the black hole. In external galaxies, kinematical information is mostly restricted to the distribution of velocities along the line of sight, so the use of either tracer implies understanding the three-dimensional velocity field. Gas disk modeling generally assumes a circular velocity field, so that deviations from Keplerian motion can give rise to errors in $M_{\mathrm{BH}}$ (e.g., Cappellari et al. 2002). Stellar kinematics are complicated by the degeneracy between the enclosed mass and the stellar orbital structure. To overcome this degeneracy, it is standard to use the full velocity profile, by either including higher order moments (van der Marel \& Franx 1993; Bender et al. 1994) or using non-parametric fitting (Gebhardt et al. 2000b). More importantly though it is essential to minimize assumptions about the stellar orbital structure. For an axisymmetric system, this generally requires orbit-based models (e.g., Gebhardt et al. 2003; van der Marel et al. 1998; van den Bosch et al. 2008). However, these models are not free of systematic concerns, such as whether to regularize (Valluri et al. 2004; van der Marel et al. 1998) or not (e.g., Gebhardt et al. 2003), ensuring adequate phase-space coverage (Valluri et al. 2004) and the possible implications of triaxiality (e.g., van den Bosch et al. 2008). Since the inclination of an elliptical galaxy is generally poorly known, this can introduce significant uncertainties into the assumed three-dimensional light distribution, and consequently 
the inferred gravitating mass (e.g., Gavazzi 2005). A reliable recovery of $M_{\mathrm{BH}}$ also depends on the accurate modeling of the total gravitating mass profile over a wide radial range, so that the results can be sensitive to the treatment of the dark matter component. Unfortunately, most published SMBH measurements from stellar dynamics omit this mass component, leading to a systematic underestimate of $M_{\mathrm{BH}}$ by as much as a factor $\sim 2$ (Gebhardt \& Thomas 2009).

Clearly, further progress demands independent verification of the different mass determination techniques. An effective way to address this question is to compare multiple $M_{\mathrm{BH}}$ measurements for the same SMBH made in different ways. However, reliable measurements of the same black hole by both dynamical methods are rare and comparisons between them yield mixed results. To date the most detailed examples are for NGC 4258 (Siopis et al. 2009; Miyoshi et al. 1995), for which the measurements were marginally discrepant (at $2.6 \sigma$, but within $\sim 15 \%$ of each other), Cen $\mathrm{A}$, for which both techniques agree within a factor $\sim 4$ but with systematic discrepancies between different measurements (even those made using the same method) that far exceed the quoted statistical errors (Silge et al. 2005; Marconi et al. 2006; Neumayer et al. 2007; Cappellari et al. 2009), and NGC 3379, for which there is general agreement for axisymmetric models (Shapiro et al. 2006; Gebhardt et al. 2000b), but not with triaxial models (van den Bosch et al. 2009) A handful of similar comparisons are less compelling due to deviations from Keplerian gas motion, or overly simple stellar modeling (e.g., Cappellari et al. 2002; Verdoes Kleijn et al. 2002).

A new way of detecting SMBHs, appropriate for earlytype galaxies, was proposed by Brighenti \& Mathews (1999), who pointed out that the gravitational influence of a quiescent SMBH on the hot, X-ray-emitting interstellar medium (ISM) should affect the temperature and density of the gas sufficiently to be detectable in Chandra observations of nearby galaxies. Although their models predict the ISM to be globally inflowing, the inflow is expected to be highly subsonic so that the ISM should remain very close to hydrostatic equilibrium, enabling $M_{\mathrm{BH}}$ to be measured directly with the standard hydrostatic X-ray mass determination methods (e.g., Gastaldello et al. 2007b; Humphrey et al. 2006, hereafter H06). If the inflow is adiabatic over scales which can be resolved with Chandra, the resulting gas compression should produce a measurable central temperature spike near to the black hole. Unlike other techniques that do not rely on dynamical modeling, such as using the variability or luminosity of an active galactic nucleus (AGN; Markowitz et al. 2003; O'Neill et al. 2005; Ferrarese \& Ford 2005 , and references therein), hydrostatic $M_{\mathrm{BH}}$ measurements do not require calibration against other methods, and their intrinsic accuracy on a case-by-case basis is limited theoretically only by the extent to which the hydrostatic approximation holds (in addition to further simplifying assumptions usually adopted to make the problem more tractable, and provided there is sufficient spatial resolution to resolve the gas properties close to the SMBH).

Since the local free-fall timescales in the centers of earlytype galaxies are generally much shorter than the gas cooling time, hydrostatic equilibrium is expected to be quickly established unless the ISM is being actively stirred up, for example by AGNs activity or galaxy merging (Mathews \& Brighenti 2003). Nonthermal pressure due to gas turbulence is not expected to perturb this equilibrium substantially; hydrodynamical simulations of structure formation suggest it contributes no more than $\sim 25 \%$ of the total pressure (Evrard et al. 1996; Nagai et al. 2007) and possibly significantly less (Fang et al. 2009). This is consistent with the general agreement between the masses of morphologically relaxed galaxy clusters measured by X-ray and gravitational lensing methods (e.g., Allen 1998; Mahdavi et al. 2008). Churazov et al. (2008) compared stellar dynamics and X-ray mass measurements in two early-type galaxies with moderately disturbed $\mathrm{X}$-ray isophotes and, similarly, found that nonthermal support contributed no more than $\sim 10 \%-20 \%$ of the global pressure. The excellent agreement between the distribution of dark matter in galaxies, groups and clusters inferred from X-ray studies and that predicted by cosmological simulations provides further corroboration of the hydrostatic approximation (Lewis et al. 2003; Vikhlinin et al. 2006; H06; Buote et al. 2007). Nevertheless, the important question of whether the gas in the galaxies studied in this paper is close to hydrostatic is one we will discuss in detail in Section 8.3.

In a recent Chandra study of the giant elliptical galaxy NGC 4649 (Humphrey et al. 2008, hereafter H08), we used this technique for the first time to constrain the mass of the SMBH. This galaxy has an extremely round, relaxed X-ray morphology, indicating little or no large-scale gas disturbance, and suggesting little turbulence that would perturb hydrostatic equilibrium by no more than a few percent (Brighenti et al. 2009). Using the $\mathrm{X}$-ray data, we constrained $M_{\mathrm{BH}}$ to $\sim 3 \times 10^{9} M_{\odot}$, which is close to the measurement from stellar dynamics (Gebhardt et al. 2003). This agreement provides compelling evidence not only that the gas must be close to hydrostatic, but also that the stellar modeling of this system is accurate, effectively ruling out pathological orbital structure.

In this paper, we extend our study to include three more objects, bringing the number of early-type galaxies in which $M_{\mathrm{BH}}$ has been determined from hydrostatic X-ray methods to a total of four. Of these, three already have $M_{\mathrm{BH}}$ determined by stellar dynamics or gas kinematics while, for the remaining galaxy, we are presenting the first direct $M_{\mathrm{BH}}$ measurement. In Section 2, we discuss the sample selection; in Section 3, we describe the X-ray data reduction; in Section 4, we discuss the deprojection of the stellar light, an essential step in modeling the mass; in Section 5, we outline how we infer the mass from the $\mathrm{X}$-ray data, and in Section 6 we report the best-fitting results. We discuss possible sources of systematic uncertainty in Section 7 and reach our conclusions in Section 8. All errors quoted in the text of this paper, unless otherwise stated, correspond to the $90 \%$ confidence regions (which, for our Bayesian analysis, implies the region of marginalized parameter space within which the integrated probability is $90 \%$ ). In the figures, we typically use $1-\sigma$ errors (68\% enclosed probability), for clarity.

\section{TARGET SELECTION}

To select our targets for this study, we searched the $H y$ perLEDA database for early-type galaxies $(T \leqslant-1)$ with heliocentric recessional velocities $<2300 \mathrm{~km} \mathrm{~s}^{-1}$ (corresponding to a distance $\sim 33 \mathrm{Mpc}$ ) and central velocity dispersion, $\sigma_{*}>280 \mathrm{~km} \mathrm{~s}^{-1}$, (corresponding to a black hole mass $\sim 5 \times 10^{8} M_{\odot}$; Tremaine et al. 2002). Restricting ourselves to gas-rich galaxies that have been observed with Chandra, our sample contained nine objects. We omitted three galaxies (M 87, NGC 3998, and IC 1459) as they contain X-ray-emitting AGNs which may make ISM temperature measurements at $\lesssim 2^{\prime \prime}$ scales challenging. This left six objects: NGC 1332, NGC 1399, NGC 4261, NGC 4374, NGC 4472, and NGC 4649. NGC 4261 also hosts an X-ray-emitting AGN but it is highly absorbed, 
Table 1

The Galaxy Sample

\begin{tabular}{|c|c|c|c|c|c|c|c|c|}
\hline Galaxy & $\begin{array}{c}\text { Distance } \\
(\mathrm{Mpc})\end{array}$ & $\begin{array}{c}L_{J} \\
\left(10^{11} L_{\odot}\right)\end{array}$ & $\begin{array}{c}\sigma_{*} \\
\left(\mathrm{~km} \mathrm{~s}^{-1}\right)\end{array}$ & $\begin{array}{l}\text { Age } \\
(\mathrm{Gyr})\end{array}$ & $\langle[Z / H]\rangle$ & Obs ID & Date & $\begin{array}{c}\text { Exposure } \\
(\mathrm{ks})\end{array}$ \\
\hline NGC 1332 & 21.3 & 0.86 & $321 \pm 14$ & $4.1_{-14}^{+8.8}$ & $0.32 \pm 0.30$ & 4372 & 2002 Sep 19 & 41 \\
\hline NGC 4261 & 29.3 & 1.4 & $308 \pm 6$ & $15 \pm 1$ & $-0.03 \pm 0.10$ & $\begin{array}{r}834 \\
9569\end{array}$ & $\begin{array}{l}2000 \text { May } 6 \\
2008 \text { Feb } 12\end{array}$ & $\begin{array}{r}34 \\
101\end{array}$ \\
\hline NGC 4472 & 15.1 & 1.7 & $294 \pm 3$ & $9 \pm 2$ & $0.17 \pm 0.12$ & 321 & 2000 Jun 12 & 34 \\
\hline
\end{tabular}

Notes. Basic data for the objects in our sample. Distances were derived from the Surface Brightness Fluctuation (SBF) Survey of Tonry et al. (2001), correcting the distance modulus by -0.16 mag to account for revisions to the Cepheid zero point (e.g., Jensen et al. 2003). Total $J$-band luminosities $\left(L_{J}\right)$ were derived from our own modeling (Section 4). The central velocity dispersion of the stars $\left(\sigma_{*}\right)$ was taken from HyperLEDA and the age and mean metallicity $(<[Z / H]>)$ were derived from our Lick index analysis (Humphrey \& Buote 2006; H06). The Chandra observation identification number (Obs ID) is given for each data set, and we list the date the observation was made and the total exposure time following removal of background flare events.

so that the thermal gas dominates the spectrum below $\sim 2 \mathrm{keV}$, enabling accurate gas temperature determination even at the smallest scales (Section 3.2; Zezas et al. 2005; H06). We excluded NGC 4374 since it has a very disturbed X-ray morphology in comparison to the other systems (Finoguenov \& Jones 2001), making accurate spectral deprojection very challenging. Although Churazov et al. (2008) argued that the gas in NGC 1399 is relatively close to hydrostatic, we also excluded that system on account of its disturbed morphology at all scales (Scharf et al. 2005) and the presence of multi-phase gas (Buote 2002; Humphrey \& Buote 2006), both of which may introduce systematic uncertainties into our measurements. We have already published the results for NGC 4649 (H08); this paper focuses on the three remaining objects (although we also discuss updated results for NGC 4649, having applied the refined analysis methods outlined in the present work). We list the basic properties of these three galaxies in Table 1.

We have previously published an X-ray mass analysis for both NGC 4261 and NGC 4472 (H06), and found that the gas appeared close to hydrostatic. However, our previous analysis focused primarily on the properties of the dark matter. Moreover, we adopted a simple, ad hoc form for the stellar mass profile, which introduced considerable systematic uncertainty into the stellar mass-to-light $(M / L)$ ratio measurement and clearly precludes the accurate determination of a central black hole mass. In this paper, we therefore present a new analysis for these galaxies, using an updated treatment of the stellar mass model, and the improved mass-determination methods outlined in $\mathrm{H} 08$. NGC 1332 is an X-ray luminous lenticular galaxy with a relaxed $\mathrm{X}$-ray morphology and a centrally peaked gas temperature profile (Buote \& Canizares 1996; Humphrey et al. 2004). Of the three galaxies, only NGC 1332 does not have a published $M_{\mathrm{BH}}$ measurement derived from dynamical methods. Both NGC 4261 and NGC 4472 have $M_{\mathrm{BH}} \sim 5 \times 10^{8} M_{\odot}$, determined from, respectively, gas kinematics (Ferrarese et al. 1996) and stellar dynamics (K. Gebhardt et al. 2009, in preparation).

\section{X-RAY DATA ANALYSIS}

\subsection{Data Reduction}

The region of sky containing each galaxy was imaged by the Chandra ACIS instrument in the ACIS-S configuration. The details of each observation are given in Table 1. The archival data were reprocessed using the CIAO 4.0 (beta 3) and Heasoft 5.3.1 software suites, in conjunction with the Chandra calibration database (Caldb) version 3.5.0. To ensure up-to- date calibration, all data were reprocessed from the "level 1" events files, following the standard Chandra data-reduction threads. ${ }^{5}$ We applied the standard correction to take account of the time-dependent gain-drift and charge transfer inefficiency, as implemented in the CIAO tools. To identify periods of enhanced background (which can seriously degrade the signal-to-noise), we accumulated background light curves for each data set from low surface brightness regions of the active chips, excluding obvious point sources. Such periods of background "flaring" were identified by eye and excised. The final exposure times are listed in Table 1. For NGC 4261, we processed both data sets independently, and then merged the final products (images, spectra, spectral response files, etc.) using the procedure outlined in $\mathrm{H} 08$, which involves correcting for relative astrometric errors by comparing positions of the detected point sources.

For each galaxy, we generated a full resolution image in the 0.5-7.0 keV energy band and a corresponding exposure map computed at an energy of $1.7 \mathrm{keV}$. Point sources were detected with the CIAO wavdetect task, which was set to search for structure at scales of $1,2,4,8$, and 16 pixels, and supplied with the exposure map to minimize spurious detections at the image boundaries. The detection threshold was set to $10^{-6}$, corresponding to $\lesssim 1$ spurious source detections per chip. All detected sources were confirmed by visual inspection, and for each appropriate elliptical regions containing approximately $99 \%$ of its photons were generated. In Figure 1, we show smoothed, flat-fielded Chandra images of each galaxy, having removed the point sources from the images. To produce these images, we first replaced all the photons within the source detection ellipse of each source with artificial data, using the algorithm described in Fang et al. (2009). The data were then flat-fielded with the exposure map and smoothed with a Gaussian kernel. Since the signal-to-noise of the image varies strongly with the distance from the center of the galaxy, the width of the Gaussian kernel was varied with the distance according to an arbitrary power law. For each galaxy, the X-ray image shows a relatively relaxed, centrally peaked morphology within the central $\sim 10 \mathrm{kpc}$ (where the stars are expected to be the dominant mass component).

In Figure 1, we overlay the radio contours from the NRAO VLA Sky Survey (NVSS) (Condon et al. 1998) on the NGC 4261 image and those from the VLA FIRST survey (Becker et al. 1995) on the image of NGC 4472. In both galaxies, the radio

\footnotetext{
5 http://cxc.harvard.edu/ciao/threads/index.html
} 


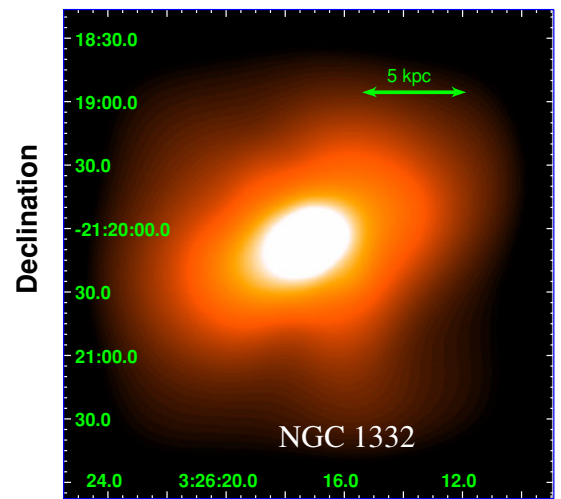

Right ascension

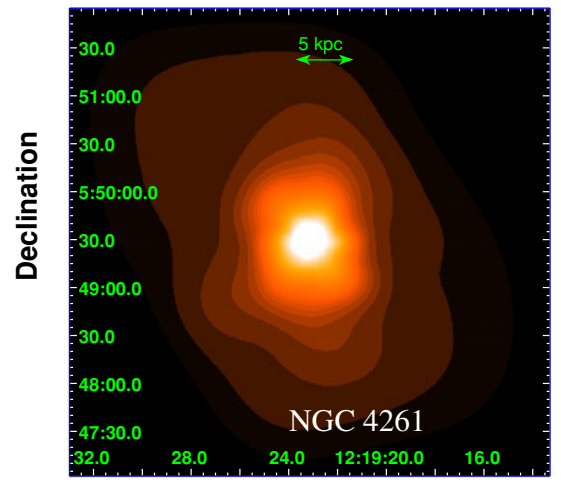

Right ascension

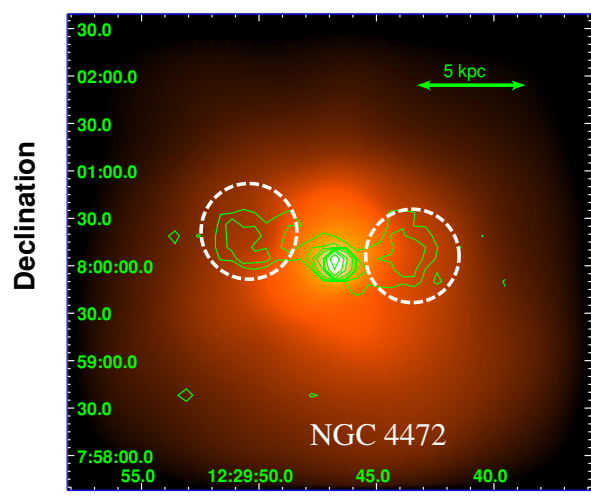

Right ascension

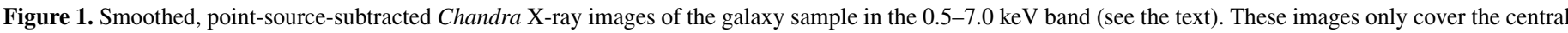

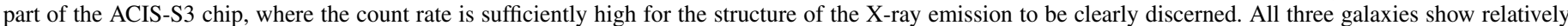

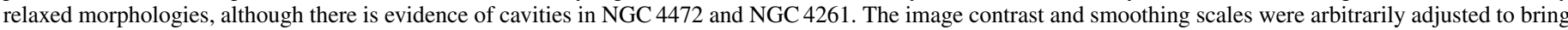

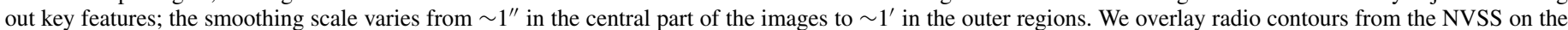

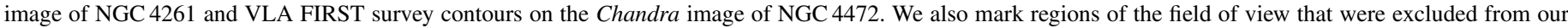
analysis (shown circumscribed by the dotted white lines).

(A color version of this figure is available in the online journal.)

lobes appear coincident with slight depressions in the X-ray brightness, which may be "cavities" (Biller et al. 2004; Croston et al. 2005). Although we have previously shown (H06) that the gas density and temperature can be fitted with physically meaningful hydrostatic solutions even if data from the vicinity of these cavities are included, to minimize potential systematic uncertainties in our subsequent analysis, we opted to mask them out, as shown in Figure 1. We also masked out data around the jet in NGC 4261 (Zezas et al. 2005).

\subsection{Spectral Analysis}

We extracted spectra in a series of concentric, contiguous annuli, placed at the X-ray centroid of each object. We determined the centroid iteratively by placing a 0.5 radius aperture at the nominal galaxy position (obtained from NED) and computing the X-ray centroid within it. The aperture was moved to the newly computed centroid, and the procedure repeated until the computed position converged. The final centroids were all within $\sim 0$.' 6 of the optical center, estimated from our HST data (Section 4), having corrected for differences in the absolute astrometry by matching X-ray and optical point sources (Humphrey \& Buote 2008).

The widths of the annuli were chosen so as to contain approximately the same number of background-subtracted photons in each (we relaxed this criterion for the innermost bins to provide as high spatial resolution as possible), and to ensure there were sufficient photons to perform useful spectralfitting. We placed a lower limit of 2 .'5 on the annulus width (for the circular central bin, this refers to its diameter) to ensure the finite instrumental spatial resolution does not lead to strong mixing between the spectra in adjacent annuli. The data in the vicinity of any detected point source were excluded, as were the data from the vicinity of chip gaps, where the instrumental response may be uncertain. We extracted products from all active chips, excluding S4 (which suffers from considerable "streaking" noise). Appropriate count-weighted spectral response matrices were generated for each annulus using the standard CIAO tasks mkwarf and mkacisrmf. For each spectrum, we estimated the background using the method outlined in $\mathrm{H} 06$.
Spectral-fitting was carried out in the energy band 0.5 $7.0 \mathrm{keV}$, using the XSPEC spectral-fitting package (Arnaud 1996). We have shown previously that fits to Poisson distributed data which minimize $\chi^{2}$ can yield significantly biased results, even if we rebin the data more than the canonical $\sim 20$ counts per bin (Humphrey et al. 2009). In contrast, we found that the C-statistic of Cash (1979) typically gives relatively unbiased results. We therefore performed the fits by minimizing $\mathrm{C}$, but nonetheless took care to estimate any residual bias on our fits by using the Monte Carlo technique outlined in Humphrey et al. (2009), as discussed in detail below. Although not strictly necessary for a fit using the C-statistic, we rebinned the data to ensure a signal-to-noise ratio $(\mathrm{S} / \mathrm{N})$ of at least 3 , and a minimum of 20 photons per bin. Such rebinning is useful during fitting as it emphasizes differences between the data and the model if the fit is poor.

For each galaxy, we fitted the data from all annuli simultaneously. This allowed us to take advantage of the projet model in Xspec, which enables spherical deprojection. Spherical symmetry is typically assumed in X-ray mass analysis, even if the $\mathrm{X}$-ray isophotes are not perfectly round, as deviations from this approximation are only expected to contribute a relatively small error into the recovered mass profile (e.g., Piffaretti et al. 2003; Gavazzi 2005). We will return to the possible impact of asphericity in a future paper; for now, however, we are interested in how accurately a conventional spherical, hydrostatic mass analysis can recover the mass profile in the inner part of the galaxy. To take account of projected emission from regions outside the outermost annulus, we used the procedure outlined in H06. To model the hot gas, we adopted a vapec component, plus a $7.3 \mathrm{keV}$ thermal bremsstrahlung component to account for undetected LMXBs (this model gives a good fit to the composite spectrum of the detected LMXBs in nearby galaxies: Irwin et al. 2003; Humphrey \& Buote 2008). For NGC 4261, we included an additional absorbed power-law component in the central bin to account for the AGN emission (H06). We adopted a slightly modified form of the Xspec vapec code to enable us to tie the ratio of each elemental abundance with respect to Fe between each annulus, while the Fe abundance $\left(Z_{\mathrm{Fe}}\right)$ was allowed to fit freely. To improve signal-to-noise, $Z_{\mathrm{Fe}}$ was tied between 

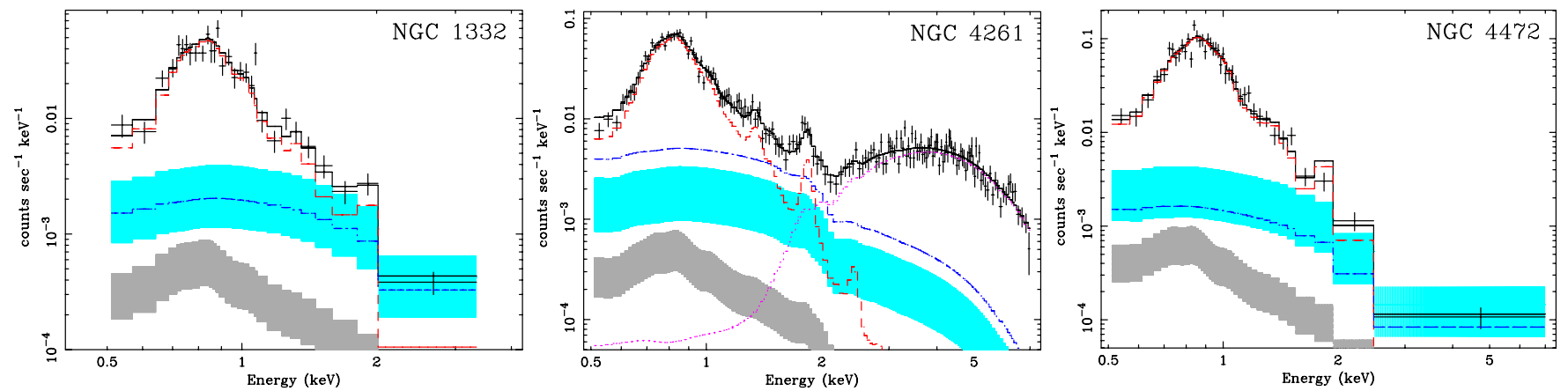

Figure 2. X-ray spectrum measured in the central bin of each galaxy. We show the measured data (without removing instrumental features), and the best-fitting model, folded through the instrumental response (solid black line). We also show individually the modeled contributions of the hot gas (dashed red line), the unresolved low-mass X-ray binaries (LMXBs; dash-dot blue line) and, for NGC 4261, the central AGN (dotted magenta line). Given known correlations between the $K$-band light and the luminosity of unresolved point sources, we show the region in which the LMXB emission is expected to lie at $1 \sigma$ uncertainty (upper, light blue shaded region), and the region in which the contribution from the composite X-ray emission of cataclysmic variables and stars is expected to lie (lower, gray, shaded region). Hot gas unambiguously dominates the spectra below $\sim 2 \mathrm{keV}$ in all three galaxies.

(A color version of this figure is available in the online journal.)

adjacent annuli, where necessary. We allowed the global ratios of $\mathrm{O}, \mathrm{Mg}$, and $\mathrm{Si}$ with respect to Fe to fit freely, while the remaining ratios were held at their Solar values (Asplund et al. 2005). For NGC 4472, we additionally freed $\mathrm{S}$ and $\mathrm{Ni}$, and for NGC 1332 and NGC 4261, we freed Ne. The absorbing column density $\left(N_{\mathrm{H}}\right)$ was fixed at the Galactic value (Dickey \& Lockman 1990). The best-fitting abundances were consistent with those reported in Humphrey \& Buote (2006) and H06.

The X-ray spectrum of the innermost bin for each galaxy is shown in Figure 2, along with the best-fitting model, folded through the instrumental responses, and the contribution of each of the spectral components. Overlaid we show the expected spectrum of the unresolved LMXBs (upper shaded region), and the expected integrated X-ray emission of cataclysmic variables and stars (lower shaded region). These were estimated from the relevant correlations with the $K$-band light reported by, respectively, Humphrey \& Buote (2008) and Revnivtsev et al. (2008). ${ }^{6}$ Since neither correlation with the $K$-band light is exact, we shade the region allowed by the $1 \sigma$ scatter in the relations. As is clear from the figure, the measured normalization of the bremsstrahlung component is consistent (within $\sim 1.8 \sigma$ ) with the LMXB flux predicted from the relation of Humphrey \& Buote (2008) for all three systems. The $K$-band magnitude was actually estimated from the I-band HST data (Section 4), and corrected for the difference in filters by using the difference in magnitudes measured between the $I$-band data and $K$-band Two Micron All Sky Survey (2MASS) data in a $\sim 7^{\prime \prime}$ aperture. Clearly, in all three galaxies, the X-ray emission in the innermost bin is completely dominated (below $\sim 2 \mathrm{keV}$ ) by the hot gas emission. This is also true for the other annuli.

Error-bars were computed via the Monte Carlo technique outlined in H06, and we carried out 100 error simulations. We show the measured kT and gas density, $\rho_{g}$ (which can be computed from the normalization of the vapec component) as a function of radius in Figure 3. As discussed in Humphrey et al. (2009), such simulations can be used to assess whether there is any bias on the best-fitting results by evaluating $f_{b}$ (the ratio of the bias to the statistic error) for each parameter.

\footnotetext{
6 Following Revnivtsev et al. (2008), for the spectrum of the CVs and stars we use a MEKAL plasma with $\mathrm{kT}=0.5$, Solar abundances relative to Anders $\&$ Grevesse (1989), plus a $\Gamma=1.9$ power-law component. We fixed the ratio of the total flux in the $2.0-7.0 \mathrm{keV}$ band to the that in the $0.5-2.0 \mathrm{keV}$ band to be 0.77 .
}

This is computed by taking the difference of the best-fitting value of each parameter and the mean value of that parameter obtained from the simulations, and then dividing this result by the statistical error. If $\left|f_{b}\right| \ll 1$, the fits are practically unbiased. In Figure 3, we show $f_{b}$ for kT and $\rho_{g}$ in each annulus. We found that, in general, $\left|f_{b}\right| \ll 1$, supporting our use of the $\mathrm{C}$-statistic. The temperature and density appeared slightly biased only in two annuli in NGC 4261, for which the error bars are large, and hence these data are unlikely to drive the temperature and density fits discussed in Section 5. The origin of the bias is unclear, but we suspect it may be related to "deprojection noise."

\section{THE STELLAR MASS DISTRIBUTION}

The modeling technique we have introduced for analyzing the X-ray data of early-type galaxies (H08) relies on an accurate (three-dimensional) representation of the stellar mass component. This is particularly true when one wishes to measure the mass of the central black hole, since one requires very accurate subtraction of the stellar mass. To determine the stellar light density, we adopted an axisymmetric deprojection technique; this is particularly important given the highly elliptical isophotes of NGC 1332, which is an almost edge-on lenticular galaxy. Although we subsequently spherically averaged the three-dimensional stellar density for use in our mass modeling (Section 5), we adopted this procedure to ensure the spherically averaged profile was as accurate as possible. Specifically, we used the iterative axisymmetric deprojection algorithm pioneered by Binney et al. (1990). This is a very general technique for axisymmetric deprojection, which is well suited for modeling isophotal shapes ranging from highly elliptical (as in NGC 1332) to very boxy (as in the central part of NGC 4261), as well as systems in which the projected isophotal ellipticity varies strongly as a function of radius, as is generally the case here (Kormendy et al. 2009; Buote \& Canizares 1996). As an axisymmetric method it cannot, however, account for the mild isophotal twists which are seen, so there will be some associated uncertainty in the deprojected light profile. Briefly, this technique involves first fitting an arbitrary function to the images, which is analytically deprojected onto an $(r, \theta)$ grid as a "first guess" for the true stellar light distribution (here the light is assumed to be axisymmetrically distributed, with coordinate $r$ indicating the distance from the center of the galaxy and $\theta$ 





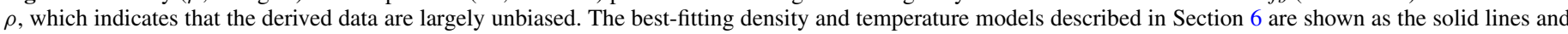

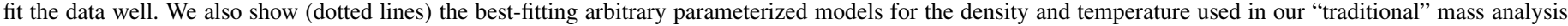
(Section 8.4). All error bars correspond to $1 \sigma$ uncertainties.

(A color version of this figure is available in the online journal.)

the zenith angle). A series of Lucy-Richardson "relaxation" iterations (Lucy 1974) subsequently improves the estimate nonparametrically.

To obtain the optical light profile, we used 2MASS $J$-band images and, to provide superior spatial resolution in the crucial inner parts of the images, HST WFPC2 data using the F814W filter (approximately $I$ band) for NGC 1332 and NGC 4472, and NICMOS2 data, using the F160W filter (approximately $H$ band) for NGC 4261. The NICMOS data were used in preference to WFPC2 data for NGC 4261 to minimize attenuation due to the central dust disk, which complicates the deprojection. Since we aimed to model the temperature and data density points measured with Chandra, for our purposes we required only the total stellar light enclosed within a region no smaller than $\sim 1^{\prime \prime}$ (Section 3.2) and it was therefore not necessary to deconvolve the instrumental point-spread function (PSF) from the HST data. The preparation of the WFPC2 data is described in Humphrey (2009), although for NGC 4472 we used different data than those listed therein (specifically, we used observation numbers $\mathrm{u} 2 \lg 0603 \mathrm{t}$ and $\mathrm{u} 2 \lg 0604 \mathrm{t}$, for which the galaxy is better centred on the PC chip). For the NICMOS data, we used the publicly available, pre-cleaned image described in Floyd et al. (2008). To prevent the emission from the weak AGN that is visible in the NICMOS image of NGC 4261 from introducing bias, we replaced the data within a central $\sim 0^{\prime \prime} .35$ region with the mean value of the surrounding pixels (which is reasonable given the flat surface brightness profile in this part of the galaxy: Kormendy et al. 2009). In practice, we did not use the entire WFPC2 and NICMOS images, but simply a portion close to the center of the galaxy, chosen to overlap the area where seeing seriously compromises the $2 \mathrm{MASS}$ data. The images are shown in Figure 4.

To obtain the initial guess for the stellar density, we simultaneously fitted models to the HST and 2MASS images us- ing custom-made software built around the MINUIT library. ${ }^{7}$ We masked out obvious point sources and the central parts of the 2MASS images, where the seeing is problematic. The WFPC2 images of NGC 1332 and NGC 4261 revealed central dusty disks; for NGC 1332, we masked this out as best we could. For NGC 4472 and NGC 4261, we were able to fit the data satisfactorily with a model which is the analytical projection of a spherically symmetric Sersic density profile (Prugniel \& Simien 1997), having broken the symmetry by arbitrarily scaling one of the coordinate axes, i.e., $\rho_{*}(x, y, z)=a \rho_{*, s}\left(\sqrt{x^{2}+(a y)^{2}+z^{2}}\right)$. Here $\rho_{*}(x, y, z)$ is the luminosity density profile, $a$ is an arbitrary scaling parameter, and $\rho_{*, s}$ is the deprojected Sersic density profile. In projection, this produces elliptical isophotes, the ellipticity of which depends on both the factor $a$ and the inclination angle $i$. In general $i$ is not known, and so we arbitrarily adopted $i=90^{\circ}$. We discuss the impact of changing the value of $i$ in Section 7. We allowed the Sersic index, effective radius, scaling factor $a$, and position angle of the isophotes to fit freely, but constrained them to be the same for both the 2MASS and HST data of a given galaxy, taking into account orientation differences between the images. The centroids of the models were allowed to fit freely in each image to allow for possible errors in the absolute astrometry. We found there were two bestfit solutions which could not be distinguished statistically-one with $a>1$ (oblate) and one with $a<1$ (prolate). By default, we adopted the oblate model, but consider the effect of using the prolate model in Section 7. For NGC 1332, the data required two such Sersic models, plus an inclined exponential disk (which can be deprojected trivially, under the assumption of an arbitrarily small but finite opening angle for the disk). We required the position angle of the Sersic models and the disk to be the same, and similarly tied the inclination of each

\footnotetext{
7 http://lcgapp.cern.ch/project/cls/work-packages/mathlibs/minuit/index.html
} 

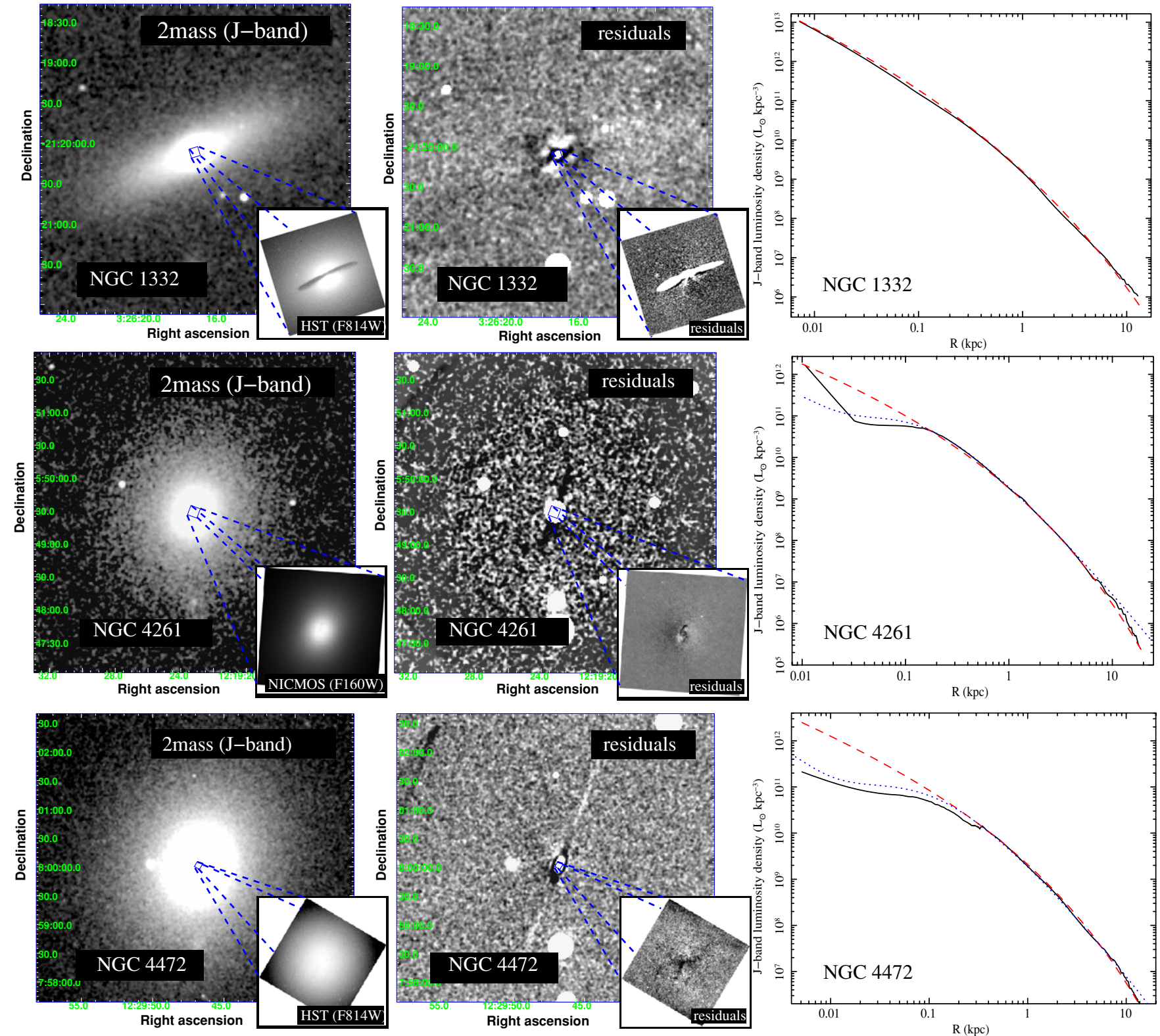

Figure 4. Left panels: $J$-band 2MASS, $I$-band $H S T$ WFPC2, and $H$-band NICMOS images of each galaxy. For the $H S T$ data, we annotate the image with the appropriate $H S T$ filter. Central panels: residual images having subtracted our projected model for the three-dimensional light distribution (see the text). Regions that were excluded from the deprojection are shown as white ellipses. Overall, these models provided a good fit to the optical light, except for the central part of NGC 4261, where residual dust extinction is problematical. Right panels: the spherically averaged luminosity density as a function of geometric radius from our model (solid lines). For NGC 4261 and NGC 4472, we overlay (dotted lines) deprojected $V$-band profiles, light profiles, arbitrarily scaled, obtained by applying the algorithm of Gebhardt et al. (1996) to the data of Kormendy et al. (2009). These profiles agree well with those obtained using our default deprojection method. We also show the best-fitting deprojected Sersic model parameterization (dashed lines) for these profiles (see the text).

(A color version of this figure is available in the online journal.)

component. Since the ellipticity of the disk model depends only on $i$, this model allowed us to constrain $i$ to $\sim 73^{\circ}$.

Having obtained the initial fits to the surface brightness profile, and hence an initial guess for the deprojected density, we used the relaxation algorithm of Binney et al. (1990) to refine our estimates. Although the algorithm they discussed is relevant for a single image, it is straightforward to generalize it to a stack of images with regions masked out. Since an image is simply a list of pixels, one always has the freedom to define a new "image," every pixel of which maps uniquely to one of the pixels in the image stack. Not every pixel in the image stack needs to have a counterpart in the new "image," so that one can readily mask out problem regions. Although this transformation complicates the definition of the projection kernel, in principle, exactly the same methods can be applied to deproject this new "image." In practice, we also subtracted the fitted background level from the count rate in each pixel before mapping it onto the new "image," resetting to zero any pixels which fall below zero counts, to prevent erroneously treating the background as a part of the source emission.

After each relaxation iteration, the "improved" density profile was projected onto the 2MASS and HST image planes. We fitted this new "model" to the data, allowing the overall normalization, the scaling factor between the HST and 2MASS data and the background levels to fit freely. We continued iterating in this manner until the reduction in the fit statistic 
on any given iteration was less than 1 , at which point we assumed convergence. In Figure 4 (middle column), we show the residuals (data minus model) obtained from our deprojection technique for each galaxy. In general, the residuals are small, except for the central arcsec of NGC 4261, where the dust disk is visible even in the NICMOS data. Due to the residual dust extinction, it seems likely that our deprojected profile for this galaxy slightly underestimates the true central stellar density; we investigate the possible impact of this underestimate in Section 7.2. In the right-hand column of Figure 4, we show the spherically averaged density profile for each system, defined as the mean luminosity density within a thin, spherical shell of given radius. We also show (dashed lines) the best-fitting spherical Sersic approximation to our deprojected profiles (the parameters of these fits are shown in the figure). These are shown to guide the eye since, by default, we use the Lucy deprojected profiles in our analysis (although in Section 7.2, we investigate how significantly our results are changed if we use the Sersic profiles shown in Figure 4). For these fits, we find $R_{e}=2.3 \mathrm{kpc}$, $3.0 \mathrm{kpc}$, and $4.2 \mathrm{kpc}$, respectively, for NGC 1332, NGC 4261 and NGC 4472, while the Sersic index, $n$, is 4.2, 3.1, and 2.9, respectively. The values of the Sersic index we obtained for NGC 4261 and NGC 4472 are lower than found by Kormendy et al. (2009), who reported values of 7.5 and 6.0, respectively, based on $V$-band surface brightness fits. The reason for the discrepancy is that we fitted the entire inner profile including the core, but did not extend the fit to large radii, while Kormendy et al. fitted out to very large radii, but excluded the core region, which they considered to be an interesting departure from the model. Over the region in which they were fitted, the models of Kormendy are formally a better fit to the data. However, we found that the lower Sersic indices obtained by us helped better capture the shape in the central few arcseconds, which are crucial for our study. Nevertheless, for our purposes the Sersic model fits are needed only to capture the global shape, rather than the fine detail, of the surface brightness profiles (Section 7.2; $\mathrm{H} 08$ ) and we do not attach any real physical significance to the particular sets of Sersic parameters we obtained.

To test further the sensitivity of our results to the deprojection method, for NGC 4261 and NGC 4472, we also used the algorithm of Gebhardt et al. (1996) to deproject the $V$-band surface brightness profiles in Kormendy et al. (2009). We assumed that the stellar isodensity surfaces can be represented by similar, axisymmetric spheroids (with axis ratios 0.75 and 0.81 for NGC 4261 and NGC 4472, respectively) and adopted an inclination angle of $90^{\circ}$. The resulting, spherically averaged density profiles are shown (arbitrarily scaled, for clarity) in the right column of Figure 4 as dotted (blue) lines. Clearly, there is good agreement with our best-fitting profiles over a wide radial range. The $V$-band profiles have a higher density outside $\sim 10 \mathrm{kpc}$, which may arise from imperfect sky subtraction in the 2MASS data. Since the stellar mass density only enters into our calculations through the total enclosed gravitating mass profile, which is dominated by the dark matter halo outside a few kpc (H06), this does not pose a significant problem for our analysis. We discuss the effect of using the deprojected Kormendy et al. profiles on our results in Section 7.2.

\section{MASS MODELLING}

The temperature and density profiles of gas in hydrostatic equilibrium are uniquely determined by four factors - the mass profile, the profile of the entropy proxy $\left(s=k T n_{e}^{-2 / 3}\right.$, where $n_{e}$ is the electron density), the gas pressure at a fiducial radius, and the total gas mass enclosed within the fiducial radius. We choose sufficiently small a fiducial radius that we can assume the enclosed gas mass to be zero. One can, therefore, invert the problem and use the temperature and density profiles to recover the mass. We have found the most robust way to achieve this is a "forward fitting" method, where physically motivated parameterized models for the mass distribution and the entropy profile are used to predict the density and temperature profiles, which are then directly fitted to the data (H08; also see Gastaldello et al. 2007b for some variations on this approach).

Assuming spherical symmetry, we modeled the enclosed mass profile as a stellar component (which was assumed to be proportional to the $J$-band luminosity enclosed in a sphere of given radius) plus a Navarro-Frenk-White (NFW; Navarro et al. 1997) dark matter component, a central supermassive black hole, and the self-consistently calculated gas mass. We allowed the stellar mass-to-light $\left(M / L_{J}\right)$ ratio to be fitted freely, along with the black hole mass $\left(M_{\mathrm{BH}}\right)$ and total mass and concentration of the dark matter halo. We modeled the entropy as a broken power law plus a constant, i.e.,

$$
s=s_{0}+s_{1} f(r),
$$

where

$$
\begin{aligned}
f(r)= & \left(\frac{r}{10 \mathrm{kpc}}\right)^{\beta_{1}} \quad\left(r<s_{\text {brk }}\right) \\
& \times\left(\frac{s_{\mathrm{brk}}}{10 \mathrm{kpc}}\right)^{\beta_{1}-\beta_{2}}\left(\frac{r}{10 \mathrm{kpc}}\right)^{\beta_{2}}\left(r \geqslant s_{\text {brk }}\right),
\end{aligned}
$$

and the parameters $s_{0}, s_{1}, \beta_{1}, s_{\text {brk }}$, and $\beta_{2}$ were parameters of the fit. Furthermore, we allowed the central gas pressure to be determined by our fit. For a more detailed description of the modeling procedure, see $\mathrm{H} 08$.

As is standard practice, we assumed that each temperature and density data-point derived from our spectral fits was a Gaussian distributed, independent random variable. This enabled us to compare the models to the temperature and density profiles by computing the $\chi^{2}$ fit statistic (since the data were not Poisson distributed, we do not expect the $\chi^{2}$ fits to be biased as described in Humphrey et al. 2009). In H08, we computed the entropy profile from the temperature and density data points, and fitted the models to the entropy and temperature profiles simultaneously. In the present work, however, we preferred to fit the models to the density and temperature as they are the quantities derived directly from the spectral fits. Clearly, this is functionally the same thing; if a model fits the temperature and density profiles well, it will also fit the entropy. For NGC 4472, we only considered data within $\sim 3.2(\sim 14 \mathrm{kpc})$ as there is evidence of X-ray asymmetries outside this range (Irwin \& Sarazin 1996; Biller et al. 2004). Although we have previously shown that reasonable hydrostatic solutions can be constructed even when including the Chandra data at larger radii (H06), we did not include those data here so as to minimize any potential source of systematic uncertainty. To obtain robust measurements of the fit parameters, rather than simply minimizing $\chi^{2}$, we adopted a Bayesian approach, as discussed below.

\subsection{The Bayesian Prior}

As with all Bayesian analysis, the choice of prior is a matter of considerable import. While for some of the fit parameters (below) this choice is natural, for other parameters (the central 
gas pressure and the parameters characterizing the entropy profile), it is less clear. In those cases, it is tempting to resort to a flat prior, but this can be problematical. Since a prior which is defined to be flat for a given parameter will no longer be flat under arbitrary transformation of that parameter (for example, taking the logarithm), even a "flat" prior adopted in ignorance arbitrarily imposes our preconceived notions on the fit. Fortunately, this choice does not always have a significant impact on the derived results; in some cases, the data may actually place much tighter constraints on the parameter than any reasonable prior, and so a flat prior is as good a default choice as any. (Formally, this is the case, if the dynamical range of the parameter allowed by the $\chi^{2}$ likelihood function is sufficiently narrow that the Jacobean introduced by a reasonable parameter transformation is approximately constant). Furthermore, if one is uninterested in the particular value of a given parameter (i.e., intending to marginalize over it), the choice of prior is also relatively unimportant provided that parameter has little or no covariance with any parameter of interest. Nevertheless, it is unlikely that one will know that the problem satisfies these criteria before actually exploring the Bayesian posterior, and so a reasonable approach is to cycle through multiple priors to examine the impact of each choice on the results. Adhering to this principle, by default, we adopted flat priors on these problematical parameters (i.e., those describing the entropy profile and the pressure at the fiducial radius), and subsequently investigated the effect of adopting alternatives (Section 7.1).

For the other parameters, the choice of prior is much clearer. A priori, we would expect that $M_{\mathrm{BH}}$ for each galaxy would agree with the $M_{\mathrm{BH}}-\sigma_{*}$ relation (Tremaine et al. 2002) and so we assumed a $\log _{10} M_{b h}$ prior, which is Gaussian distributed about this relation, given the intrinsic scatter in this relation (taken to be $0.25 \mathrm{dex}$ ) and the error on $\sigma_{*}$ (Table 1). We discuss the sensitivity of our results to the form of this relation in Section 7.1. Additionally, we have also explored the effect of using a flat prior for $M_{\mathrm{BH}}$ which, although poorly motivated, is useful for disentangling to what extent the measured constraints are due to the prior and due to the data (Section 6). ${ }^{8}$

Given the measured age and mean abundance of the stellar population in each galaxy (Table 1), stellar population synthesis models can be used to predict the stellar $M / L_{J}$ and its uncertainty (Section 8.2). Although this provides a natural prior for $M / L_{J}$, it is of interest to compare the population synthesis predictions to those obtained from the fits. Therefore, to avoid circular reasoning, we instead used a flat prior for $M / L_{J}$, but confirmed that consistent results were obtained with the more physical choice (Section 7.1). In practice $M / L_{J}$ is sufficiently well constrained by the data that this choice has little effect on the results.

If the galaxies were chosen entirely at random (irrespective of morphological type or central velocity dispersion), one might simply adopt the halo mass function predicted by dark matter simulations as the prior on the virial mass of the dark matter halo.

\footnotetext{
8 We note that another possible choice here, that of a prior which is flat on $\log M_{\mathrm{BH}}\left(\right.$ which is the same as $\operatorname{Prob}\left(M_{\mathrm{BH}}\right)=1 / M_{\mathrm{BH}}$ in linear space), would be a poor choice, since it would imply a priori that $M_{\mathrm{BH}}$ is as likely to be found in, for example, the range $10^{3}-10^{4} M_{\odot}$ as the range $10^{8}-10^{9} M_{\odot}$. Since the data for two of the galaxies have little discriminating power at very low masses (Figure 8), the use of this prior would unrealistically skew the black hole masses low and, crucially, will be sensitive to the lower mass cutoff. Conversely, a prior which is flat in $M_{\mathrm{BH}}$ produces a posterior probability distribution that is closer to that produced with the (more realistic) $M_{\mathrm{BH}}-\sigma_{*}$ prior. Since the data tightly constrain the upper limit on $M_{\mathrm{BH}}$, the results using the flat prior are relatively insensitive to the upper mass cutoff.
}

However, this is clearly not appropriate here; a better choice would be to restrict the allowed range of the total baryon fraction within the virial radius, based on the predictions of a theoretical model, as was done in H06. Unfortunately, the physics of feedback is highly uncertain, making the results then sensitive to the assumptions implicit in the adopted model. Alternatively, one could employ an empirical relation linking the mass of the galaxy to its host halo (e.g., Conroy \& Wechsler 2009). We investigate this option in Section 7 but, in the interest of more generality in our results, by default we used a uniform prior for the logarithm of the halo mass in the range $\log _{10} M_{d m}=10^{12}$ $10^{14} M_{\odot}$. For a prior on the concentration, we adopted the theoretical concentration-halo mass relation for relaxed halos found by Macciò et al. (2008). We used their relation for the "WMAP1" $\Lambda$ CDM (cold dark matter) cosmology since, of the cosmologies they considered, that is most consistent with observations of galaxies, groups and clusters (Buote et al. 2007).

\subsection{Exploration of the Bayesian Posterior}

The exploration of the Bayesian posterior for a multiparameter model is typically carried out with Monte Carlo methods; we employed the publicly available ${ }^{9}$ MultiNest code version 1.0 (Feroz et al. 2008; Feroz \& Hobson 2008), which implements a robust and efficient nested sampling algorithm (a Monte Carlo integration technique optimized for these purposes: Skilling 2004). We have verified that we obtained consistent results with a Metropolis-algorithm-based Markov Chain Monte Carlo code. Since nested sampling integrates over a finite volume, in the absence of hard boundaries for any parameters (such as the restricted range of dark matter virial masses we considered), we restricted each parameter to $\mathrm{a} \pm 10-\sigma$ region about the "best-fitting" value. This was estimated by first minimizing $\chi^{2}$ with the Levenberg-Marquardt algorithm (e.g., Press et al. 1992), which provides for each parameter both the "best-fitting" value and an estimate of the statistical error, $\sigma$. On completion of the nested sampling run, we checked for self-consistency between the initial estimates of $\sigma$ and the final error bars. If the latter were significantly larger than the initial guess, we re-ran the nested sampling algorithm with our refined error estimate, thus ensuring at least a $\sim \pm 10 \sigma$ region of integration for each parameter.

On completion, the nested sampling algorithm returns a set of data points which sample the posterior reasonably well. One can use these data to construct a probability density histogram for each parameter, marginalizing over the other parameters. From this histogram, we found both the most probable values and a $90 \%$ confidence region, defined by stepping out along lines of constant probability density until $90 \%$ of the total probability was enclosed. We found that the results do not depend sensitively on the width of the histogram used.

\section{RESULTS}

The model described in Section 5 was able to fit the density and temperature profiles well, and the best-fitting models are shown in Figure 3. Strikingly, we do not see a sharp central temperature spike arising from the gravitational influence of the black hole, similar to that previously reported for NGC 4649. There is a modest temperature rise in the center of NGC 1332, but this is mostly a consequence of the peaked stellar density profile and is a feature of our models even if $M_{\mathrm{BH}}=0$. Of

\footnotetext{
9 http://www.mrao.cam.ac.uk/software/multinest/
} 
Table 2

Fit Results

\begin{tabular}{ccccccccc}
\hline \hline Galaxy & $\chi^{2} /$ dof & $\begin{array}{c}M_{\mathrm{BH}} \\
\left(10^{9} M_{\odot}\right)\end{array}$ & $\begin{array}{c}M / L_{*} \\
\left(M_{\odot} L_{\odot}^{-1}\right)\end{array}$ & $\begin{array}{c}s_{0} \\
\left(\mathrm{keV} \mathrm{cm}^{2}\right)\end{array}$ & $\begin{array}{c}s_{1} \\
\left(\mathrm{keV} \mathrm{cm}^{2}\right)\end{array}$ & $\beta_{1}$ & $\begin{array}{c}s_{\text {brk }} \\
(\mathrm{kpc})\end{array}$ & $\beta_{2}$ \\
\hline NGC 1332 & $11.9 / 8$ & $0.52_{-0.28}^{+0.41}$ & $1.16_{-0.14}^{+0.12}$ & $0.55_{-0.31}^{+0.31}$ & $33.3_{-4.6}^{+4.9}$ & $0.969_{-0.098}^{+0.12}$ & $\ldots$ & $\ldots$ \\
& & $(0.49)$ & $(1.15)$ & $(0.39)$ & $(34.4)$ & $(0.951)$ & $\ldots$ & $\ldots$ \\
NGC 4261 & $22.9 / 12$ & $0.44_{-0.24}^{+0.28}$ & $1.621_{-0.092}^{+0.071}$ & $0.586_{-0.097}^{+0.12}$ & $77.6_{-8.2}^{+8.7}$ & $1.325_{-0.066}^{+0.066}$ & $13.4_{-3.5}^{+9.4}$ & $0.47_{-0.31}^{+0.21}$ \\
& & $(0.48)$ & $(1.627)$ & $(0.558)$ & $(78.6)$ & $(1.332)$ & $(13)$ & $(0.54)$ \\
NGC 4472 & $22.1 / 14$ & $0.64_{-0.33}^{+0.61}$ & $1.473_{-0.11}^{+0.085}$ & $1.3_{-0.37}^{+0.25}$ & $66.1_{-8.4}^{+11}$ & $1.31_{-0.13}^{+0.1}$ & $5.23_{-0.76}^{+1}$ & $0.67_{-0.16}^{+0.11}$ \\
& & $(0.69)$ & $(1.486)$ & $(1.23)$ & $(66.7)$ & $(1.3)$ & $(5.32)$ & $(0.63)$ \\
\hline
\end{tabular}

Notes. The fit results for each galaxy, adopting the $M_{\mathrm{BH}}-\sigma_{*}$ prior for $M_{\mathrm{BH}}$. We show $\chi^{2} / \mathrm{dof}$ for the best-fit model (Section 6) and, for each interesting parameter, the most probable value and $90 \%$ confidence regions, marginalized over the other parameters. Since the set of marginalized values is not necessarily the set of parameter values which maximizes the posterior, we also list in parentheses the parameter values which correspond to such a "best fit."

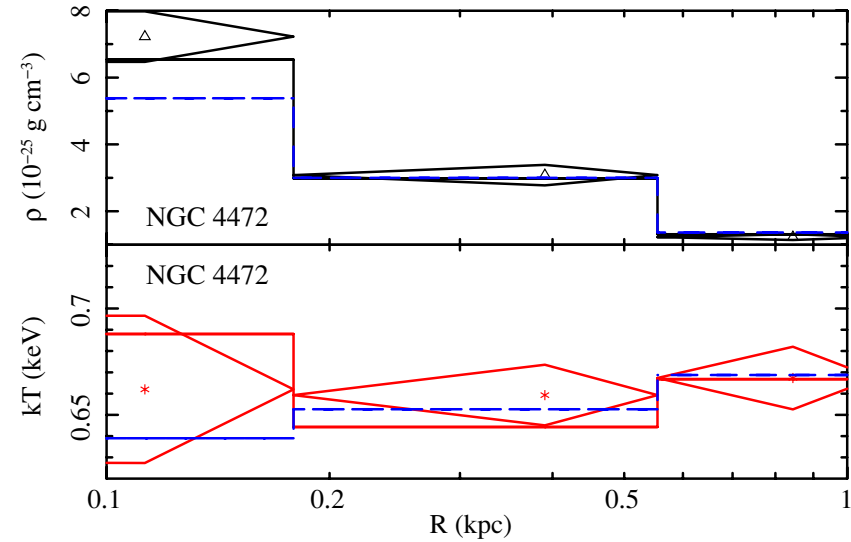

Figure 5. Central part of the temperature and density profiles for the gas in NGC 4472, illustrating the effect of adding a black hole. We show the bestfitting model with a nonzero $M_{\mathrm{BH}}$ (solid lines) and the best-fitting model with $M_{\mathrm{BH}}=0$. The latter model is a poorer fit to both profiles in the central bin; the difference in $\chi^{2}$ between the two models is 5.5. Error bars correspond to $1 \sigma$ uncertainties.

(A color version of this figure is available in the online journal.)

all the galaxies, only our model fits to NGC 4472 show any evidence of a higher central temperature in the presence of the black hole, and even then the effect is very weak, as shown in Figure 5 (which compares the best-fitting temperature and density models with and without a central black hole). We discuss the reasons for this difference from NGC 4649 in Section 8.1.

The interesting marginalized parameters from our fit are given in Table 2. For each object, the dark matter halo mass and concentration were degenerate with each other, and we could not place interesting constraints on the virial mass (at least for NGC 1332 and NGC 4472) without applying an additional prior, such as a restriction on the allowed baryon fraction (see H06). Rather than imposing such an ad hoc constraint, since neither of the dark matter halo parameters strongly correlate with $M_{\mathrm{BH}}$ or $M / L_{J}$, we simply marginalized over them and do not report them here. For NGC 1332, we were able to fit the data using a steep $\left(\beta_{1}=0.96\right)$ entropy profile without any evidence of a break; we could not constrain $s_{\text {brk }}$ and so we fixed it to a large value, outside the field of view. For NGC 4261 and NGC 4472, the entropy profiles were similarly steep in the center of the galaxy, but flattened outside $\sim 5-10 \mathrm{kpc}$. We show the entropy data points, derived from the temperature and density data points, plus the best-fitting entropy models in Figure 6. This characteristic shape for the entropy profile is similar to that found in recent observations of X-ray bright galaxy groups (Jetha et al. 2007; Finoguenov et al. 2007; Gastaldello et al. 2007a; Sun et al. 2009), but $s_{\text {brk }}$ is at a comparatively smaller scale.

The measured radial distribution of total gravitating mass is shown in Figure 7 for each galaxy, along with the contribution from each of the separate mass components. We found that the stellar mass component dominates within $\sim 5-10 \mathrm{kpc}$, with a significant contribution of dark matter required to explain our observations at larger radii in NGC 1332 and NGC 4261 (for NGC 4472, the data we fitted did not extend to large enough radii for the influence of the dark matter to be easily discerned). These results are consistent with our previous analysis for NGC 4261 (and NGC 4472), despite the much simpler stellar mass profiles used in that work (H06).

Since the stellar mass is non-negligible in the innermost bin, the constraints on $M_{\mathrm{BH}}$ for these galaxies are less tight than we were able to obtain for NGC 4649. Unfortunately, this makes the results depend, to some degree, on the choice of prior for $M_{\mathrm{BH}}$. In Figure 8, we compare the marginalized probability density functions for $M_{\mathrm{BH}}$ for the case where the $M_{\mathrm{BH}}-\sigma_{*}$ is used as a prior and for the flat prior case with the probability density function of the $M_{\mathrm{BH}}-\sigma_{*}$ prior itself. For NGC 4472 , there is a clear peak in the probability density when the flat prior is applied, allowing $M_{\mathrm{BH}}$ to be constrained to $M_{\mathrm{BH}}=\left(1.04_{-0.76}^{+0.62}\right) \times 10^{9} M_{\odot}(90 \%$ confidence $)$, and excluding $M_{\mathrm{BH}}=0$ at $98 \%$ probability (or " $2.3 \sigma$ "). ${ }^{10}$ Applying the $M_{\mathrm{BH}}-\sigma_{*}$ relation as a prior causes the probability density peak to shift and narrow somewhat, but our conclusions are not qualitatively changed. For NGC 1332 and NGC 4261, the prior has a more significant effect on the results; however, below $\sim 3 \times 10^{8} M_{\odot}$, the $\left(M_{\mathrm{BH}}-\sigma_{*}\right)$ prior completely dominates the shape of the probability density function, while the X-ray data largely determine its shape above $\sim 4 \times 10^{8} M_{\odot}$. Put another way, the X-ray data alone provide a firm upper limit for $M_{\mathrm{BH}}$ for these two galaxies, while the $M_{\mathrm{BH}}-\sigma_{*}$ relation eliminates the portion of parameter space in which $M_{\mathrm{BH}} \rightarrow 0$, which is inconsistent with the apparent ubiquity of SMBHs in giant early-type galaxies.

In Figure 9, we compare our constraints on $M_{\mathrm{BH}}$ for each galaxy with measurements using other methods. For NGC 4472, we compare to the stellar dynamical measurement of K. Gebhardt et al. (2009, in preparation; omitting a dark matter halo), for NGC 4261 we use the gas disk dynamics measurement of Ferrarese et al. (1996), and for NGC 1332, for which there is

\footnotetext{
${ }^{10}$ This result is also obtained with a simple frequentist analysis: the difference in $\chi^{2}$ between the best-fitting model and the best-fitting model with $M_{\mathrm{BH}}=0$ is 5.5 (which corresponds to a $98 \%$ confidence region). On the basis of an $f$-test, the significance of the black hole detection is therefore $\sim 94 \%$.
} 

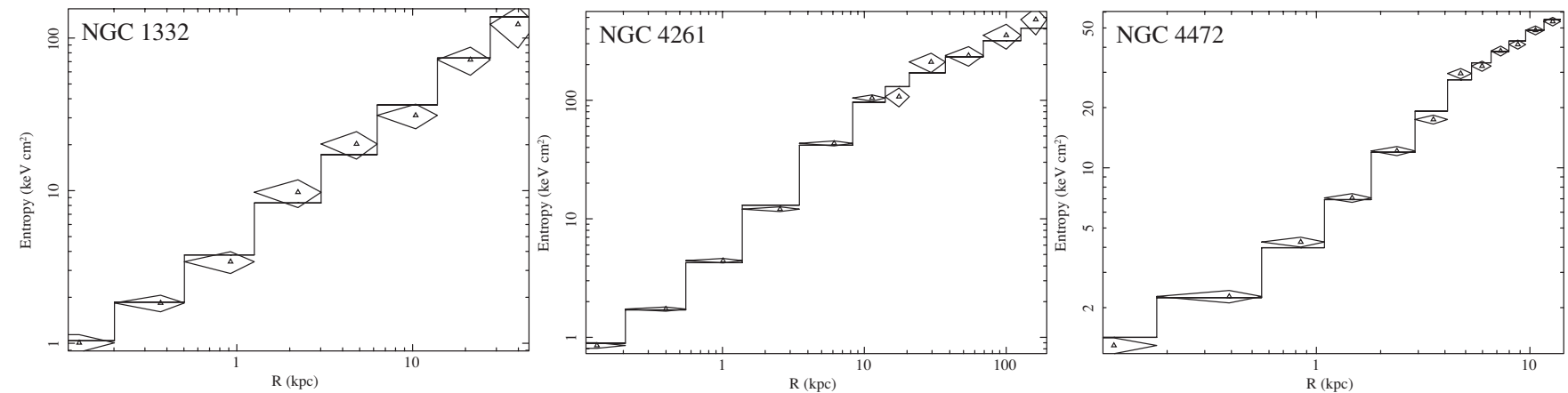

Figure 6. Entropy profiles for each galaxy, derived from the density and temperature data in Figure 3. We also show the best-fitting entropy models (Section 6), which fit the data very well. Error bars correspond to $1 \sigma$ uncertainties.
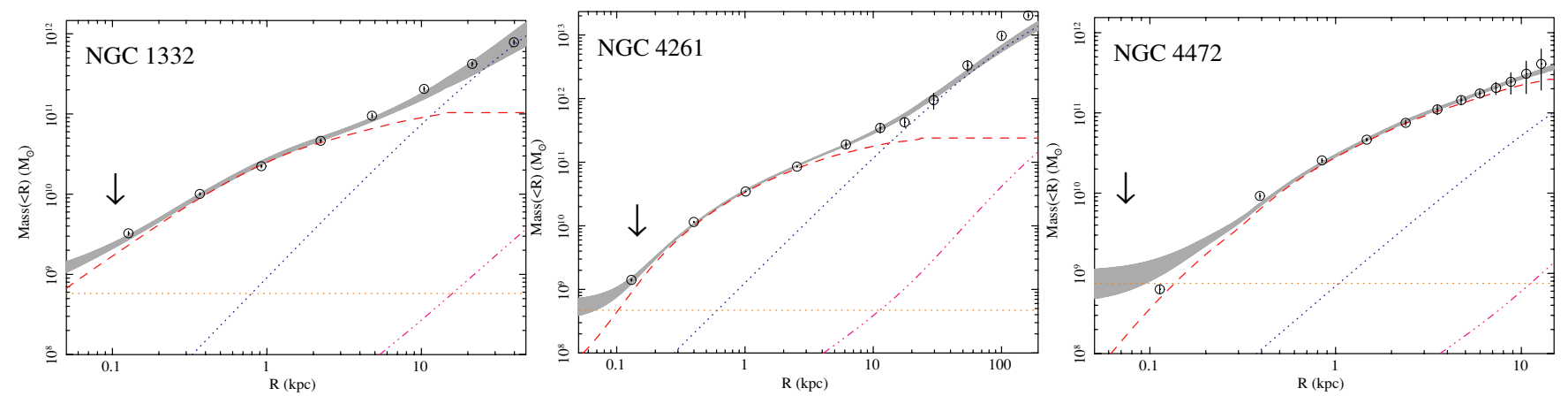

Figure 7. Radial mass profiles for each galaxy, derived from our fits to the Chandra data. The solid (black) lines indicate the total enclosed mass, the dashed (red) lines indicate the stellar mass, the dotted (blue) lines were the dark matter contribution, the dash-dot (orange) lines are the black hole mass, and the dash-dot (magenta) line is the gas mass contribution. The gray shaded regions indicate the $1 \sigma$ error on the total mass distribution. Overlaid are a set of data points (and $1 \sigma$ errors) derived from a more "traditional" mass analysis (Section 8.4), which generally agrees very well with the fitted models (we stress the models are not fitted to these data points, but are derived separately). The arrows indicate the spatial scale corresponding to $1^{\prime \prime}$.

(A color version of this figure is available in the online journal.)
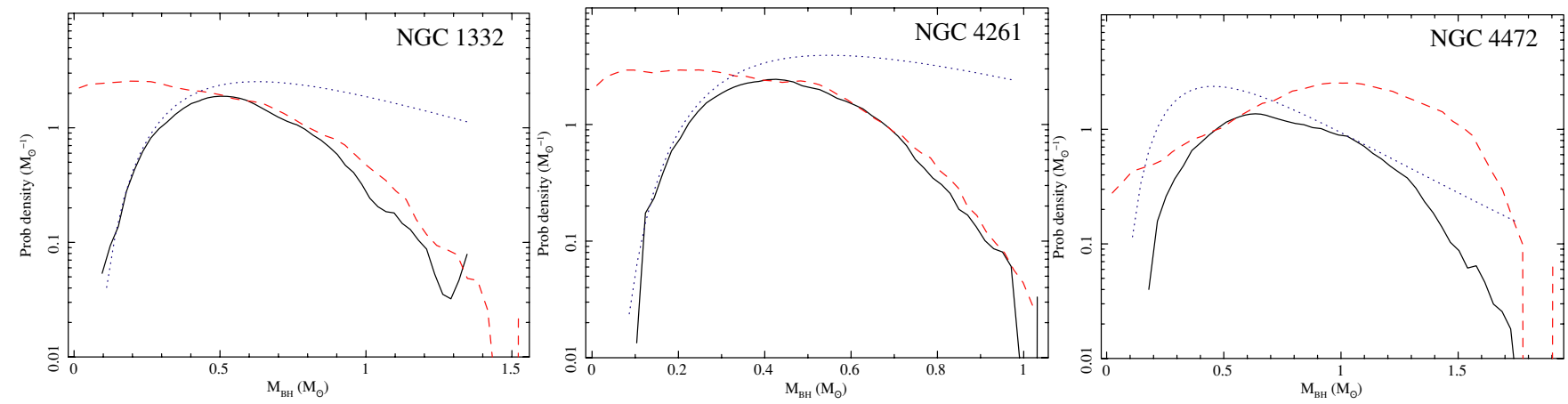

Figure 8. Marginalized probability density distributions for $M_{\mathrm{BH}}$ for each galaxy. We show, as a solid line, the probability density when the $M_{\mathrm{BH}}-\sigma_{*}$ relation was used as a prior. The dashed (red) lines are the probability density function with a flat prior, and the dotted (blue) lines are the $M_{\mathrm{BH}}-\sigma_{*}$ relation prior itself; both have been arbitrarily scaled for clarity. Also for clarity, we smoothed the distribution functions with a fourth-order Savitzky-Golay filter, which spans $\sim 20 \%$ of the $M_{\mathrm{BH}}$ range for each system. For NGC 1332 and NGC 4261, the shape of the probability density function below $\sim 3 \times 10^{8} M_{\odot}$ is mostly determined by the prior, while above $\sim 4 \times 10^{8} M_{\odot}$, the constraints are mostly given by the X-ray data. The X-ray data alone provide only upper limits for these two objects. For NGC 4472, the shape of the probability density function is much less sensitive to the prior.

(A color version of this figure is available in the online journal.)

no published dynamical $M_{\mathrm{BH}}$ measurement, we simply compare to $M_{\mathrm{BH}}$ estimated from the $M-\sigma$ relation. We also include the results for a re-analysis of NGC 4649, applying the same Bayesian methods and stellar light modeling used in the present paper (and the stellar dynamical analysis of Shen \& Gebhardt 2009). For completeness, we show the results using both choices of prior on $M_{\mathrm{BH}}$. As expected, for NGC 4472 and NGC 4649, the constraints are relatively insensitive to this choice. There is clearly agreement between the X-ray and stellar dynamical mass measurements. Particularly striking is that, for NGC 4649, the error bars obtained from the X-ray method are roughly half the size of those found by the stellar dynamical modeling. The $\mathrm{X}$-ray measurement for NGC 4472 is marginally higher than the dynamically determined $M_{\mathrm{BH}}$. However, in the revised analysis of NGC 4649 by Shen \& Gebhardt (2009), $M_{\mathrm{BH}}$ increased by a factor $\sim 2$ from that obtained by Gebhardt et al. (2003). Whether this increase is due to inclusion of a dark matter halo in the newer models or improved dynamical modeling will require additional studies. Nevertheless, it is plausible that there could be a similar revision for NGC 4472 when the dark matter halo is included, which would bring it into even better agreement with the X-ray data. 

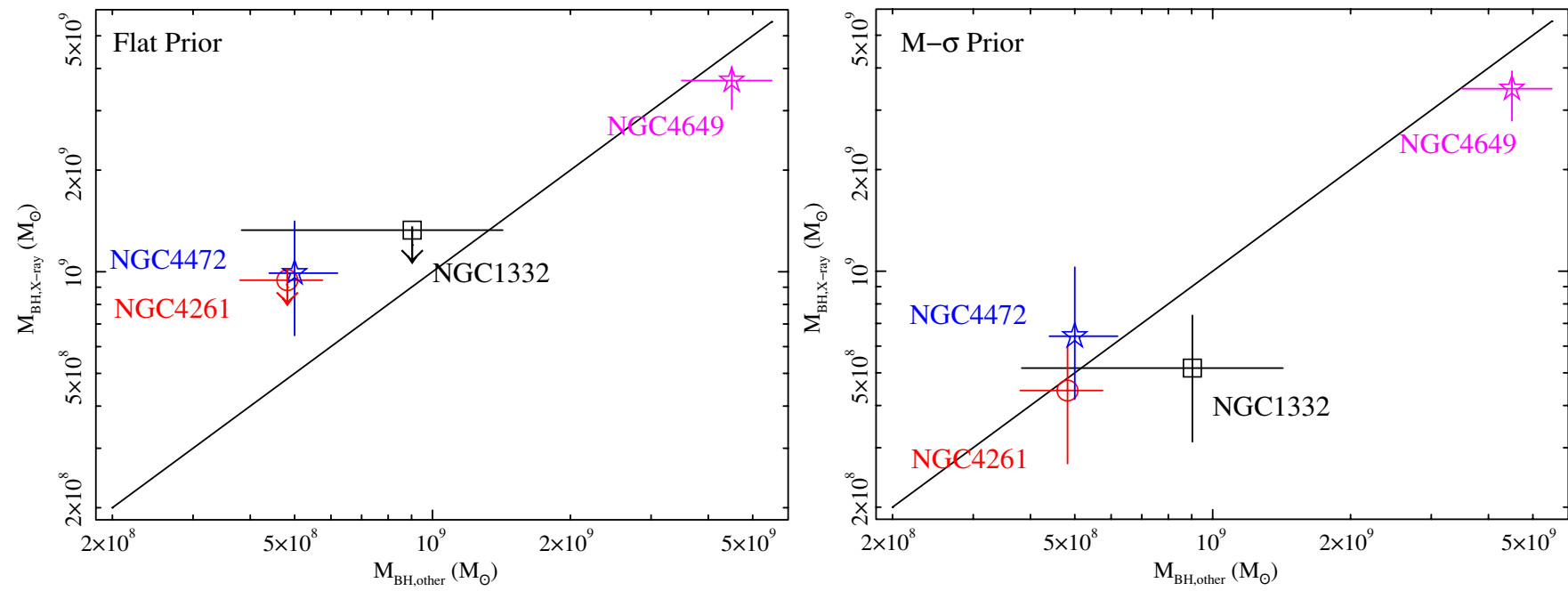

Figure 9. Left panel: the derived black hole mass $\left(M_{\mathrm{BH}}\right)$ from the X-ray data, assuming a flat prior for $M_{\mathrm{BH}}$, shown vs. black hole determinations from stellar dynamics (for NGC 4472 and NGC 4649; stars), gas disk dynamics (NGC 4261; circle) or the $M-\sigma$ relation (NGC 1332; square). Error bars correspond to $1 \sigma$ uncertainties, although for NGC 1332 and NGC 4261, we show the $3 \sigma$ upper limit (marked as an arrow). The dotted line indicates $y=x$. Right panel: the same, but using the $M_{\mathrm{BH}}-\sigma_{*}$ relation as a prior on $M_{\mathrm{BH}}$. Note that the comparison of the NGC $1332 M_{\mathrm{BH}}$ measurement using this prior with the $M_{\mathrm{BH}}-\sigma_{*}$ relation, as shown in the right panel, should be treated with caution. We find good agreement between the masses measured with the different techniques.

(A color version of this figure is available in the online journal.)

For NGC 4261, the data unambiguously rule out that $M_{\mathrm{BH}}$ determined from X-rays substantially exceeds the gas disk dynamical measurement. Although the lower limit from our analysis is largely determined by the prior (so we cannot place stringent limits on any bias due to nonthermal pressure support, although see Section 8.1), there is no evidence from our analysis of a discrepancy between the gas disk dynamics measurement and our X-ray results. For NGC 1332, our results constitute the first direct measurement of the SMBH mass, and so in Figure 9, we compare it to the $M_{\mathrm{BH}}-\sigma_{*}$ relation rather than a measurement derived from stellar dynamics. We find consistency; the X-ray determined $M_{\mathrm{BH}}$ clearly does not lie significantly above this relation.

\section{SYSTEMATIC ERROR BUDGET}

In this section, we address the sensitivity of our results to various data analysis choices that were made, including the choice of prior. In most cases, it is difficult or impractical to express these assumptions through a single additional model parameter over which one might hope to marginalize, and so we adopt the pragmatic approach of investigating how our results change if the assumptions are arbitrarily adjusted. We focused on those systematic effects likely to have the greatest impact on our conclusions, and list in Table 3 how the "most probable," marginalized $M_{\mathrm{BH}}$ and $M / L_{J}$ measurements for each galaxy are affected by each test. These estimates constitute a likely upper limit on the magnitude of each systematic uncertainty and we stress they should not be added in quadrature with the statistical errors. We outline below how each test was performed; so those readers uninterested in the technical details of our analysis may wish to proceed directly to Section 8 .

\subsection{Priors}

As outlined in Section 5.1, the choice of priors on several of the parameters is arbitrary. We therefore considered the effect of replacing each arbitrary choice with another, reasonable prior. Specifically, for each parameter describing the entropy profile, and for the central gas pressure, we switched from a flat prior on the parameter to a flat prior on its logarithm. The effect, summarized in Table 3 as $\Delta$ Prior(entropy), is typically smaller than the statistical errors. Similarly, we have experimented with replacing the flat prior on $M / L_{J}$ with a Gaussian prior, the mean and sigma of which are determined from the stellar population synthesis models; see Section 8.2. The effect of this choice is summarized as $\Delta \operatorname{Prior}(M / L)$ in Table 3. To assess the importance of the $M_{\mathrm{vir}}$ prior, we experimented with instead fixing the $M_{\text {vir }}$ to a canonical value, based on the empirical relation of Conroy \& Wechsler (2009), which links the virial mass of a halo to the stellar mass it hosts. We computed the stellar mass from the $L_{J}$ values given in Table 1 and the bestfitting stellar $M / L_{J}$ ratio for each galaxy. The effect of this choice is summarized as $\Delta \operatorname{Prior}\left(M_{\mathrm{vir}}\right)$. Finally, we investigated the effect of our $M_{\mathrm{BH}}$ prior by adopting the revised and updated $M_{\mathrm{BH}}-\sigma_{*}$ relation for early-type galaxies reported by Gültekin et al. (2009) as the prior. The results ( $\Delta$ Prior(Gultekin)) indicate that this has little effect on our conclusions. In addition, we also report the result of using the (poorly motivated) flat prior on $M_{\mathrm{BH}}\left(\Delta\right.$ Prior(Flat $\left.M_{\mathrm{BH}}\right)$ ), as discussed in Section 5.1. In general, adopting the flat prior had a larger impact on the derived parameters than any of the other choices.

\subsection{Stellar Model}

As shown in H06, careful modeling of the stellar light is necessary to obtain an accurate measurement of the mass-tolight ratio. Similarly, since we did not typically resolve scales at which the black hole is completely dominating the gravitating mass, we also expect $M_{\mathrm{BH}}$ to be sensitive to this modeling. For NGC 4261 and NGC 4472, both of which have uncertain geometry, we experimented with using a prolate model instead of an oblate model, and changing the inclination from $90^{\circ}$ to $45^{\circ}$. In NGC 1332, the geometry is less uncertain, since the presence of the disk allowed us constrain the inclination and symmetry axis. The effect of these choices is listed as $\Delta \operatorname{Stars}$ (geometry) in Table 3.

In Section 4, we compared our deprojected stellar density profiles to those obtained by using the deprojection method 
Table 3

Systematic Error Budget

\begin{tabular}{|c|c|c|c|c|c|c|}
\hline \multirow[t]{2}{*}{ Test } & \multicolumn{2}{|c|}{ NGC 1332} & \multicolumn{2}{|c|}{ NGC 4261} & \multicolumn{2}{|c|}{ NGC 4472} \\
\hline & $\begin{array}{c}M_{\mathrm{BH}} \\
\left(10^{9} M_{\odot}\right)\end{array}$ & $\begin{array}{c}M / L_{J} \\
\left(M_{\odot} L_{\odot}^{-1}\right)\end{array}$ & $\begin{array}{c}M_{\mathrm{BH}} \\
\left(10^{9} M_{\odot}\right)\end{array}$ & $\begin{array}{c}M / L_{J} \\
\left(M_{\odot} L_{\odot}^{-1}\right)\end{array}$ & $\begin{array}{c}M_{\mathrm{BH}} \\
\left(10^{9} M_{\odot}\right)\end{array}$ & $\begin{array}{c}M / L_{J} \\
\left(M_{\odot} L_{\odot}^{-1}\right)\end{array}$ \\
\hline Most Probable & $0.52_{-0.28}^{+0.41}$ & $1.16_{-0.14}^{+0.12}$ & $0.44_{-0.24}^{+0.28}$ & $1.62_{-0.09}^{+0.07}$ & $0.64_{-0.33}^{+0.61}$ & $1.47_{-0.11}^{+0.09}$ \\
\hline$\Delta$ Prior(entropy) & \pm 0.04 & \pm 0.02 & $\begin{array}{l}+0.09 \\
-0.04\end{array}$ & -0.01 & +0.17 & -0.02 \\
\hline$\Delta \operatorname{Prior}(M / L)$ & -0.04 & -0.03 & -0.13 & +0.07 & +0.05 & -0.02 \\
\hline$\Delta \operatorname{Prior}\left(M_{\mathrm{vir}}\right)$ & +0.11 & -0.01 & +0.04 & +0.00 & +0.24 & -0.02 \\
\hline$\Delta$ Prior(Gultekin) & -0.04 & -0.03 & -0.10 & +0.00 & +0.10 & +0.01 \\
\hline$\Delta$ Prior(Flat $\left.M_{\mathrm{BH}}\right)$ & -0.27 & +0.00 & -0.16 & +0.01 & +0.35 & -0.02 \\
\hline$\Delta$ Stars(geometry) & $\ldots$ & $\ldots$ & -0.09 & -0.19 & $\begin{array}{l}+0.08 \\
-0.01\end{array}$ & -0.11 \\
\hline$\Delta$ Stars(deprojection) & $\ldots$ & $\ldots$ & -0.02 & -0.14 & -0.22 & -0.24 \\
\hline$\Delta$ Stars(Sersic) & -0.09 & -0.13 & -0.22 & -0.06 & -0.15 & -0.15 \\
\hline$\Delta \mathrm{DM}$ & -0.09 & +0.07 & -0.07 & +0.10 & +0.07 & +0.09 \\
\hline$\Delta$ Background & +0.09 & -0.14 & +0.02 & -0.06 & -0.16 & +0.08 \\
\hline$\Delta N_{\mathrm{H}}$ & -0.07 & +0.03 & -0.03 & +0.01 & \pm 0.03 & $\begin{array}{l}+0.03 \\
-0.02\end{array}$ \\
\hline$\Delta \mathrm{XRB}$ & -0.10 & +0.04 & \pm 0.02 & -0.02 & -0.15 & \pm 0.02 \\
\hline$\Delta$ Plasma code & -0.08 & +0.03 & -0.01 & -0.06 & +0.25 & +0.04 \\
\hline$\Delta$ Bandwidth & -0.11 & \pm 0.03 & -0.04 & -0.02 & $\begin{array}{l}+0.33 \\
-0.16\end{array}$ & \pm 0.07 \\
\hline$\Delta$ Statistic & -0.06 & -0.01 & -0.06 & +0.00 & +0.01 & -0.05 \\
\hline$\Delta$ Centroid & +0.08 & -0.12 & $\ldots$ & $\ldots$ & +0.02 & +0.00 \\
\hline$\Delta$ Distance & \pm 0.02 & \pm 0.09 & -0.07 & $\begin{array}{l}+0.17 \\
-0.15\end{array}$ & -0.03 & \pm 0.06 \\
\hline$\Delta$ Rotation & -0.08 & +0.09 & -0.05 & +0.01 & +0.01 & -0.01 \\
\hline
\end{tabular}

Notes. Estimate of the likely impact of potential sources of systematic errors in our analysis on our measurements of $M_{\mathrm{BH}}$ and the stellar $M / L_{J}$. We give the marginalized value and $90 \%$ statistical errors for each galaxy (top line). For a series of tests (see the text), the change in the marginalized value caused by an arbitrary change of the underlying assumptions in our analysis is given. We stress that these systematic errors should not be added in quadrature with the statistical errors. In most (but not all) cases, the systematic errors are no larger than the statistical errors.

of Gebhardt et al. (1996), finding general consistency within the central $\sim 10 \mathrm{kpc}$. Nevertheless, to quantify any systematic uncertainties associated with the deprojection method, we also used the $V$-band profiles for NGC 4261 and NGC 4472. Given the different filters, we corrected the $M / L$ ratios obtained based on the colors measured in a $\sim 30^{\prime \prime}$ aperture, taking the $V$-band data from Sandage \& Visvanathan (1978) and computing the $J$-band magnitudes from the 2MASS data discussed in Section 4 . We found that use of this profile did not lead to significantly different conclusions ( $\Delta$ Stars(deprojection) in Table 3 ). For all three galaxies, we also investigated the effect of adopting a simple Sersic approximation for the stellar light, as shown in Figure 4. This is useful as residual dust extinction in the center of NGC 4261 likely leads us to underestimate the stellar density, so that the true density profile lies intermediate between the preferred deprojected profile and the simple Sersic parameterization. The impact of this test is listed as $\Delta$ Stars(sersic) in Table 3. All three tests had a noticeable effect on the best-fitting results, comparable to the measured statistical errors. Nevertheless, these sources of uncertainty should not qualitatively affect our conclusions.

\subsection{Dark Matter Halo}

Although we showed in H06 that the dark matter halos of a sample of early-type galaxies were consistent with NFW profiles obeying the concentration-virial mass relation predicted by numerical structure formation simulations (see also Buote et al. 2007), in some recent stellar dynamical analyses, it has been argued that a "cored logarithmic" profile may provide a better fit (e.g., Thomas et al. 2007). Therefore, to test the extent to which the modeling of the dark halo affects our results, we experimented with using the cored logarithmic profile in place of NFW. We found this had a slight effect (summarized as $\triangle \mathrm{DM}$ in Table 3), comparable to the other sources of error. We will return to the question of the optimal shape of the dark matter halo in a future paper.

\subsection{Spectral Modeling}

We investigated several potential sources of systematic uncertainty in our spectral analysis. First, we considered the impact of our background treatment, by adopting the spectra derived from the standard background template events files distributed with the CIAO Caldb, having renormalized them to match the count rate in the 9-12 keV band. This choice had a measurable effect on our results, comparable to the statistical uncertainties ( $\Delta$ Background in Table 3 ). We also investigated how our results are affected by changing $N_{\mathrm{H}}$ by $\pm 25 \%\left(\Delta N_{\mathrm{H}}\right)$, replacing the bremsstrahlung component with a $\Gamma=1.5$ power law or adjusting its temperature by $\pm 25 \%(\triangle \mathrm{XRB})$, using a MEKAL rather than an APEC plasma ( $\triangle$ Plasma Code), or changing the energy range over which the fitting was performed to $0.4-7.0 \mathrm{keV}$, $0.5-2.0 \mathrm{keV}$ or $0.7-7.0 \mathrm{keV}$ ( $\Delta$ Bandwidth). Two further tests we carried out were using the $\chi^{2}$ fit statistic rather than the C-statistic ( $\Delta$ Statistic), which had only a very small effect, and moving the centroid of the extraction regions by up to $\sim 0^{\prime \prime} .5$, consistent with the accuracy we estimate for our centroiding procedure ( $\Delta$ Centroid). This latter test was not carried out for NGC 4261, since the X-ray detection of the AGN makes the centroiding very accurate.

\subsection{Other Tests}

Two final issues we investigated were the uncertainty in the adopted distance to the galaxies, and the possibility of gas rotation. For the former, we adjusted the distance by the $1 \sigma$ errors reported in Tonry et al. (2001), and the effect is 
summarized as $\Delta$ Distance in Table 3 . To test the impact of the latter, we allowed the gas to rotate along with the stars and included an additional nonthermal pressure component to the equation of hydrostatic equilibrium, following Fang et al. (2009). We consider this to be likely an upper limit on the gas rotation; in none of the galaxies do we find the X-ray isophotes are much flatter than the optical light, as might be expected if the gas is rapidly rotating (for a more detailed discussion, see Brighenti et al. 2009). For NGC 1332, we adopted the major axis rotation profile from Dressler \& Sandage (1983), for NGC 4261, we used the minor axis profile from Bender et al. (1994), and for NGC 4472 we used the major axis profile of Sánchez-Blázquez et al. (2007). Assuming that the gas rotates as a rigid body, we find (unsurprisingly) that rotation increases the amount of enclosed mass we measure, but the effect ( $\Delta$ Rotation) is not larger than the statistical errors on $M / L_{J}$ or $M_{\mathrm{BH}}$.

\section{DISCUSSION}

\subsection{Black Hole Mass Measurements}

Under the assumptions of hydrostatic equilibrium, spherical symmetry, a single-phase ISM and a stellar mass component which follows the optical light, we have constrained the masses of the SMBHs at the centers of three galaxies. Combined with our previous measurement for NGC 4649 (H08), this demonstrates that hydrostatic X-ray methods are a practical, competitive means for SMBH mass determination. We found that the masses determined from the X-ray method are in good agreement with those found from other techniquesspecifically stellar kinematics for NGC 4472 and NGC 4649 (K. Gebhardt et al. 2009, in preparation; Gebhardt et al. 2003) gas disk dynamics for NGC 4261 (Ferrarese et al. 1996), and the $M_{\mathrm{BH}}-\sigma_{*}$ relation for NGC 1332. This makes NGC 4261, NGC 4472, and NGC 4649 three of only a small handful of galaxies which have $M_{\mathrm{BH}}$ determined by more than one method.

The agreement between our results and the SMBH masses obtained from other methods (especially for NGC 4472 and NGC 4649, for which the constraints do not strongly depend on the prior) provides support for the assumptions in our analysis. Key among these assumptions is that of hydrostatic equilibrium. While one might expect deviations from this approximation to be most prevalent close to the SMBH, since the ejecta from accretion episodes are known to interact with the ISM at large scales, the local free-fall timescale is shortest in the central region of the galaxy so that hydrostatic equilibrium will quickly be re-established if the gas is stirred up. Furthermore, the physics of the coupling between an AGN and the surrounding ISM remains very uncertain, particularly on such small scales. We would expect deviations from hydrostatic equilibrium most likely to manifest themselves in nonthermal pressure support (e.g., Churazov et al. 2008; Zappacosta et al. 2006), leading the X-ray method to underestimate the true $M_{\mathrm{BH}}$. As is clear from Figure 9, the X-ray measurement for NGC 4472 is slightly higher than the measurement from stellar dynamics, while those for NGC 4649 are very close, suggesting that any such nonthermal pressure is small. While, for NGC 1332 and NGC 4261, there is also no evidence that the X-ray-determined mass is systematically underestimated (since the upper limits imposed by the X-ray data are consistent with the gas disk measurement for NGC 4261 and the $M_{\mathrm{BH}}-\sigma_{*}$ relation for NGC 1332), the data do not allow us to rule this out, since the lower limit on $M_{\mathrm{BH}}$ is largely determined by the prior. To investigate this in more detail there is a clear need for higher quality Chandra data, which should allow more stringent upper limits to be placed on $M_{\mathrm{BH}}$. Since the $M_{\mathrm{BH}}-\sigma_{*}$ relation is not sufficiently accurate for this purpose, this will also require an accurate $M_{\mathrm{BH}}$ determination from stellar dynamics for NGC 1332. Despite these concerns for these two systems, it is unlikely that any deviations from hydrostatic equilibrium are restricted solely to the central bin, and deviations at larger radius should produce residuals in our fit to the temperature and density profiles and a systematically underestimated stellar $M / L_{J}$ ratio. As we discuss in Section 8.3, the good fits to these profiles at larger radii, combined with reasonable $M / L_{J}$ ratios for each galaxy in fact suggest that hydrostatic equilibrium is a reasonable approximation.

Another crucial assumption in our analysis is that the ISM is approximately single phase at any radius. Although this approximation is not exact for NGC 4472, as evinced by regions of cool gas in its center (Caon et al. 2000), the good overall agreement between $M_{\mathrm{BH}}$ determined from the X-rays and optical methods strongly suggests that it is, nevertheless, fairly accurate (implying a small filling factor for the cool phase). The dusty central disks in NGC 1332 and NGC 4261 similarly reveal departures from a perfectly single phase ISM. While, as discussed above, we cannot rule out the possibility that the $\mathrm{X}$-rays may underestimate the mass for these two systems, provided the filling factor of the cool phase is small (for example, if the gas is restricted to this disk-like geometry), the density and pressure of the hot phase obtained from our X-ray measurements will be fairly accurate, and thus sufficiently accurate for our purposes.

The agreement we found between the $M_{\mathrm{BH}}$ measurements not only provides strong support for our X-ray approach but also crucial, independent verification that the dynamical $M_{\mathrm{BH}}$ measurement techniques are accurate. As discussed in Section 6, the stellar dynamical measurements for NGC 4472 and NGC 4649 are marginally lower than $M_{\mathrm{BH}}$ obtained from the X-rays, but this could arise from the omission of the dark matter halo in the stellar modeling (e.g., Shen \& Gebhardt 2009). Notwithstanding, this comparison suggests that deviations from triaxiality, uncertainties in the inclination of the galaxy, and pathological orbital structure cannot give rise to additional systematic errors larger than a factor $\sim 2$ in $M_{\mathrm{BH}}$ for either galaxy (unless they are compensated for by finely tuned systematic errors in the X-ray determined mass). The similar agreement for NGC 4261 also suggests that any systematic errors in the $M_{\mathrm{BH}}$ determination are not significantly larger than the current statistical errors. This is interesting since Ferrarese et al. (1996) used sparsely sampled HST FOS data, while higher quality STIS data suggest that non-Keplerian motions may be nonnegligible (Noel-Storr et al. 2003). Since the prior dominates the probability density function at low masses, the agreement we find partially reflects the fair agreement between the gas disk measurement of NGC 4261 and the $M_{\mathrm{BH}^{-}}-\sigma_{*}$ relation of Tremaine et al. (2002), which was derived in part from this data point. Still, it is very unlikely that this one point strongly influenced either the shape or scatter of their fit, so, combined with the tight upper limit on the mass from the X-ray datawhich is also consistent with this datum - the agreement is very encouraging.

In NGC 4649, the primary effect of the SMBH on the gas was to introduce a sharp central temperature peak, similar to that predicted by Brighenti \& Mathews (1999). However, none of the three galaxies in the present study shows such a pronounced effect. This difference in behavior can be understood in part as a consequence of the black hole mass. In NGC 4649, the SMBH 
dominates the mass profile for much of the region enclosed by the central bin. Technically, the stellar mass enclosed within this bin is larger, but what is more important is the mass evaluated not at the outer radius of the bin, but at an appropriate intermediate position (see e.g., Gastaldello et al. 2007b). Using an appropriate radius, the black hole actually dominates the mass, contributing $77_{-8}^{+3} \%$ of the total (see Figure 7 ), whereas in the other galaxies $M_{\mathrm{BH}}$ is almost an order of magnitude lower and the stellar mass largely dominates (formally, it contributes $18 \% \pm 10 \%, 35 \% \pm 15 \%$, and $65_{-22}^{+10} \%$ of the mass in the central bin for NGC 1332, NGC 4261 and NGC 4472, respectively). Correspondingly, therefore, the SMBH has a smaller influence on the gas. Another key difference between NGC 4649 and the other galaxies is the shape of its entropy profile, which is very flat within $\sim 500 \mathrm{pc}$, in contrast to the approximately $s \propto R$ profiles at small scales shown in Figure 6. Since a lower central entropy tends to reduce the temperature of the gas in the center, any temperature peak is suppressed in NGC 4472, NGC 4261, and NGC 1332.

One possible concern with our analysis is the dependence of the best-fitting $M_{\mathrm{BH}}$ measurements on the $M_{\mathrm{BH}}-\sigma_{*}$ relation, which we used as a prior. In particular, the $M_{\mathrm{BH}}-\sigma_{*}$ relation is determined in large part from stellar dynamical measurements which do not include dark matter halos. Since this omission is able to bias the measured $M_{\mathrm{BH}}$, the exact shape or scatter in this relation could be in error, particularly at the critical high-mass end, which may have an impact on our measured parameters. However, if significant, we would expect this to revise upward the mass expected from the prior, which would serve only to restrict further the parameter space allowed (Figure 8) and shrink our error bars. Moreover, we obtained qualitatively the same results when the flat prior was used in place of the $M_{\mathrm{BH}}-\sigma_{*}$ relation (albeit we then only obtained upper limits on $M_{\mathrm{BH}}$ for two of the four galaxies), giving us confidence that subtle revisions to the $M_{\mathrm{BH}}-\sigma_{*}$ relation will not change our conclusions. Still, for a precision comparison of $M_{\mathrm{BH}}$ determined by different techniques, and, in particular, constraining the $M_{\mathrm{BH}}-\sigma_{*}$ relation itself from X-ray data alone, deeper Chandra observations will be useful as higher quality data generally result in reduced sensitivity to the priors.

Although we have demonstrated that hydrostatic X-ray mass analysis is potentially a powerful means to determine $M_{\mathrm{BH}}$ in gas-rich early-type galaxies, such measurements are contingent upon spatially resolved X-ray spectroscopy at the smallest possible scales. Given the limitations of Chandra, it seems likely that this technique will only be applicable to a handful more galaxies in the near future (Section 2). However, with the spatial resolution promised by future X-ray missions, in particular Generation-X (Windhorst et al. 2006), such measurements should become routine. A factor $\sim 5$ improvement in the PSF would likely correspond to $\sim 2$ orders of magnitude increase in surveyable volume and hence number of accessible systems. Further, with such spatial resolution, it will become possible routinely to resolve the sphere of influence of the SMBH, making the $M_{\mathrm{BH}}$ measurement no longer dependent primarily on one data bin.

\subsection{Stellar $M / L_{J}$ Ratios}

In addition to the $M_{\mathrm{BH}}$ measurements, our hydrostatic models also enabled us to constrain the stellar $M / L_{J}$ ratios, under the assumption that the stellar mass follows light. In Figure 10, we compare our measured values to those predicted by three sets of single-burst stellar population (SSP) models, given the

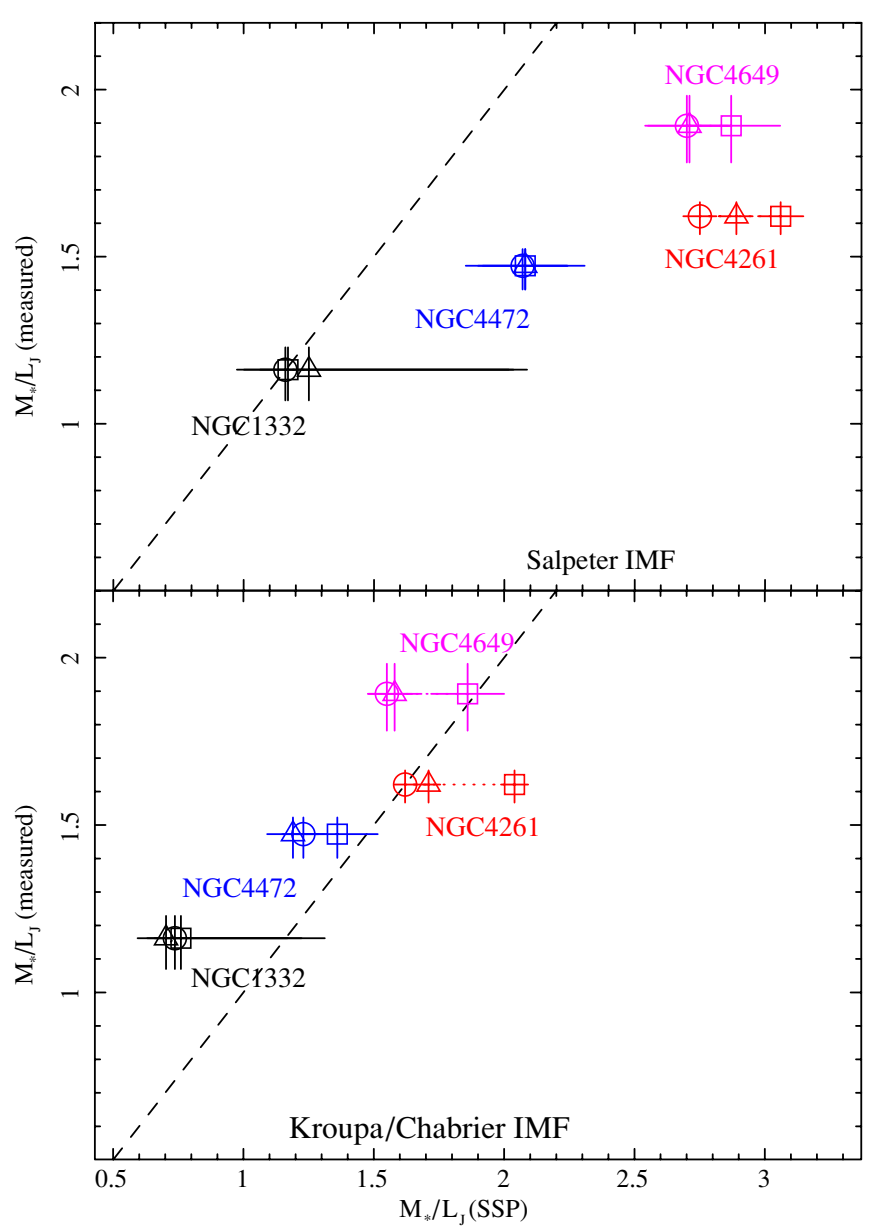

Figure 10. Comparison of the stellar $M / L_{J}$ ratios determined from our X-ray analysis to the predictions of a selection of SSP models. Squares denote the SSP models of Maraston (2005), circles denote PEGASE (Fioc \& Rocca-Volmerange 1997) models, and triangles denote the models of Bruzual \& Charlot (2003). In the upper panel, we show the synthetic SSP $M / L_{J}$ ratios computed for a Salpeter IMF and in the lower panel, we use the Kroupa (2001) IMF for the Maraston and PEGASE models, and the Chabrier (2003) IMF for the Bruzual $\&$ Charlot models. The dotted line denotes the $y=x$ relation. We find good overall agreement between the measured $M / L_{J}$ ratios and the models using a Kroupa or Chabrier IMF, suggesting that the gas is close to hydrostatic.

(A color version of this figure is available in the online journal.)

stellar age and abundance listed in Table 1. In particular, we used the models of Maraston (2005, using the updated model grids made available by the author ${ }^{11}$ ), the PEGASE code version 2 (Fioc \& Rocca-Volmerange 1997), and the models of Bruzual \& Charlot (2003). With the Maraston and PEGASE models, we investigated both the Salpeter initial stellar mass function (IMF) and that of Kroupa (2001), while for the Bruzual \& Charlot models we used the Salpeter and the Chabrier (2003) IMFs, and the "Padova 1994" evolutionary tracks. To compute the predictions of the SSP models, we linearly interpolated $M_{*} / L_{J}$ from the synthetic values tabulated on a convenient grid as a function of metallicity and age. The observed $M / L_{J}$ we reported in $\mathrm{H} 08$ for NGC 4649 is 20\% lower than shown here, which arises due to the improved modeling of the stellar light in the present paper; the galaxy was assumed to be spherical in H08, whereas it is appreciably flattened outside a few kpc (Peletier et al. 1990), which we take into account with the deprojection method outlined in Section 4.

\footnotetext{
11 http://www.dsg.port.ac.uk/ marastonc. At the metallicity of these galaxies, the differences between the models with a blue and red horizontal branch are negligible.
} 
Although there is considerable scatter in the synthetic $M / L_{J}$ values predicted by the SSP models, we find good overall agreement between the predictions of the models using a Kroupa or Chabrier IMF and the results of our fits. In contrast, the models using the Salpeter IMF dramatically overestimate $M / L_{J}$. While this general trend is consistent with the conclusions of our previous work, in H06 our measured $M / L$ ratios were typically significantly smaller even than those assuming a Kroupa IMF. In particular, for NGC 4472 , we measured $M / L_{K}$ to underestimate the SSP value by $\sim 40 \%$. The origin of this discrepancy, which has vanished in our updated analysis, can be easily traced to the simpler form for the stellar density profile used in our earlier work. In H06, we modeled the stellar light as a Hernquist (1990) profile, which significantly differs from the density profile derived in Section 4 outside $\sim 4 \mathrm{kpc}$. This difference was exacerbated by our not choosing the parameters for the stellar model in a self-consistent manner, thus introducing considerable additional systematic error into our older $M / L_{K}$ measurement (see Section 7.2 of H06).

While the agreement in the lower panel of Figure 10 is striking, it is unlikely that real early-type galaxies are monolithic, single-burst stellar populations. Indeed, the presence of line strength and color gradients (e.g., Peletier et al. 1990; Trager et al. 2000) indicates that the metallicity may vary radially and at least some elliptical galaxies host multiple-aged stellar populations (e.g., Rembold et al. 2005). Fitting SSP models to the Lick indices of such a system in order to determine a mean age and metallicity may yield a biased result, although if one population dominates the stellar light, the bias may be small. Such a bias is most problematical for the age, which is the most important factor influencing $M / L_{J}$ for an old system. Older populations typically have larger $M / L_{J}$ ratios and so, since the mean stellar ages we estimated for NGC 4649 and NGC 4261 are both $\gtrsim 13 \mathrm{Gyr}$, the "true" $M / L_{J}$ for these systems cannot be higher than our SSP predictions, for a given IMF. While this could in principle allow the X-ray data to be reconciled with a Salpeter IMF, for these two systems this would require the stellar light to be largely dominated by stars aged less than $\sim 8 \mathrm{Gyr}$, implying a very large bias on the measured age. Given the good agreement for all the systems between the measured $M / L_{J}$ ratios and the SSP models computed for a Kroupa or Chabrier IMF, a simpler hypothesis is that the bias is actually small and the Salpeter IMF is not correct.

If the stellar population properties do vary radially, this can cause a violation of the assumption that the stellar mass strictly follows the light. Nevertheless, in the very red photometric band, we adopt here the effect of varying the metallicity is very slight (e.g., significantly changing the metallicity of a $\sim 10$ Gyr population, assuming a Kroupa 2001 IMF and the SSP models of Maraston 2005, from $[\mathrm{Fe} / \mathrm{H}]=0.35$ to -0.33 causes only a $\sim 6 \%$ change in the stellar $M / L_{J}$ ratio), so a metallicity gradient is not a concern. Furthermore, if an age gradient is present which might significantly affect the $M / L$ ratio, it is likely to be give rise to a substantial color gradient. In practice, all four galaxies show only very slight evidence for any such gradient, at least in $V-I$ color (Poulain 1988; Poulain \& Nieto 1994, see also the good agreement between the shapes of the deprojected $V$-band and $J$-band profiles shown in Figure 4 for NGC 4261 and NGC 4472). Therefore, the assumption that stellar mass follows $J$-band light is likely to be reasonable.

We note that simple models of galaxy formation suggest that the condensation of gas into stars should produce a gravitational reaction on the dark matter halo, leading to a cuspier dark matter profile through a process termed "adiabatic contraction" (Blumenthal et al. 1986; Gnedin et al. 2004). Such models increase the total gravitating mass in the center of the galaxy substantially, thus lowering the stellar mass-tolight ratio one measures for a given object (H06) when the models are applied. Our data clearly leave little room for a substantial reduction in the measured $M / L_{J}$ ratios if we require consistency with SSP models. This is in clear contradiction with the adiabatic contraction models as currently implemented, unless they operate in a finely tuned conspiracy with nonthermal pressure (Section 8.3), an IMF radically different from those discussed here, or a mixture of multiple-aged stellar populations in each galaxy. This result is not surprising since similar difficulties for the adiabatic contraction models have been reported for disk galaxies (Dutton et al. 2007; Gnedin et al. 2007) and recent simulations have found baryonic condensation to a have a significantly smaller effect on the dark matter halo (Abadi et al. 2009).

Finally, it is interesting to compare our measured stellar mass to light ratios with those obtained from stellar dynamical methods. Unfortunately, most dynamical studies to date have involved significant simplifications, such as omitting a dark matter component, assuming spherical symmetry or limiting the generality of the orbital structure, which may prevent a fair comparison with our work. Of those incorporating a dark matter halo, Kronawitter et al. (2000) constructed spherical dynamical models for NGC 4472, but restricted the phase-space distribution by applying an expansion using a set of known basis functions. They found $M / L_{B} \sim 8-10$, correcting to our adopted distance, which corresponds to $M / L_{J} \sim 2.4$ 3 , assuming a $B-J$ color for this galaxy of 3.1 (from NED), which is larger than our best-fitting $M / L_{J}$. However, the blue band they adopted is more sensitive to any contamination by a young stellar population, so that the stellar mass may not follow B-band light as faithfully as the $J$-band light. Furthermore, the simplifications in their modeling and the use of a logarithmic (rather than an NFW) dark matter halo potential (which can affect the best-fitting $M / L$; Section 7.3) make the implications unclear. More general axisymmetric, orbit-based models that incorporate a dark matter halo (also using the logarithmic potential) have been constructed for NGC 4649 by Shen \& Gebhardt (2009), who obtained $M / L_{V}=8.0 \pm 0.9 M_{\odot}$ (roughly $\sim 70 \%$ higher than our measurement, when correcting for the galaxy color). The authors were not able, however, to explore fully a range of inclination angles, which could affect the recovered $M / L_{V}$ ratio (e.g., Gavazzi 2005; Krajnović et al. 2005). Still, we discuss the possible implications of this discrepancy in Section 8.3.

\subsection{Hydrostatic Equilibrium}

Our results suggest that the hot ISM of these galaxies must be close to hydrostatic. Strong, highly localized deviations from this approximation would likely manifest themselves as significant residuals in our fits to the density and temperature profiles, which are not generally seen (Figure 3). More global deviations would result in a systematically misestimated pressure and consequently a bias in the measured enclosed mass, which is not borne out by the good agreement between the measured stellar $M / L_{J}$ ratios and the predictions of SSP models for a reasonable IMF (Section 8.2). The agreement we find between our $M_{\mathrm{BH}}$ measurements and those from different techniques supports the hydrostatic approximation extending into the central parts of the 
galaxies (albeit less emphatically on account of the larger error bars).

In practice, the selection of galaxies which are not significantly morphologically disturbed in the X-ray, at least over the scales under scrutiny (excepting possible cavities, which we excluded from our analysis), eliminated galaxies in which hydrostatic equilibrium is most likely perturbed. Still, deviations from hydrostatic equilibrium could persist which do not obviously disturb the image, due to bulk or turbulent gas flows, magnetic fields or cosmic rays. Each of these effects will likely contribute nonthermal pressure support, causing X-ray mass techniques to underestimate the true mass (e.g., Nagai et al. 2007; Zappacosta et al. 2006; Churazov et al. 2008; Fang et al. 2009). If such nonthermal pressure were prevalent in these galaxies it must be compensated for by errors in the SSP models themselves, which must underestimate the mass by almost the same amount. As discussed in Section 8.2, at least for two of the galaxies, NGC 4261 and NGC 4649, this cannot be achieved simply by allowing a complex star-formation history, and requires a modification of the IMF from the Kroupa or Chabrier form. Adiabatic contraction, or a similar effect, would also increase the gravitating mass in the central part of the galaxy and thus could offset the effects of such putative nonthermal pressure without requiring a modification of the IMF. In either case, however, the magnitude of the effect would have to be, serendipitously, balanced with the nonthermal pressure.

Taking at face value the $M / L$ ratios inferred from the PEGASE SSP models that use a Kroupa IMF (which, of all the tested models, agree best with the measurements), one can place an upper limit on the average global fraction of the pressure which is nonthermal $(\alpha)$ by fitting a model of the form

$$
M / L_{J}(\text { observed })=(1-\alpha) M / L_{J}(\mathrm{SSP}) .
$$

To take into account the errors on both $x$ - and $y$-axes, we used a procedure similar to that outlined in Press et al. (1992). We obtained a marginally acceptable fit $\left(\chi^{2} / \mathrm{dof}=7.4 / 3\right)$ with $\alpha<0.02$ ( $3 \sigma$ confidence region), indicating that no more than a few percent of the pressure support could be due to deviations from hydrostatic equilibrium. More conservatively, we estimated the effect of inherent errors in the models by cycling through the results for the three different SSP codes. While this does not account for the possibility of a complex star formation history, the failure to account for that effect would mean that $\alpha$ is overestimated (at least for NGC 4261 and NGC 4649, which provide crucial high $M / L_{J}$ leverage). The largest allowed value of $\alpha$ was $\sim 0.18$ (at $3 \sigma$ significance) for Maraston's models.

Assuming the SSP models are correct, these constraints on nonthermal pressure are particularly important since two of the galaxies, NGC 4261 and NGC 4472, host active AGNs and associated radio lobes that have carved out cavities in the ISM. The very small value of $\alpha$ dramatically illustrates that the mere existence of cavities does not immediately imply that the ISM is out of hydrostatic equilibrium in regions away from the cavities (which were excluded from our analysis). Such stringent limits on deviations from hydrostatic equilibrium are consistent with the similar $\sim 10 \%-20 \%$ limit reported by Churazov et al. (2008) for two galaxies which are manifestly more disturbed than our sample. Theoretical structure formation models which produce morphologically relaxed-looking objects similarly suggest no more than $\sim 25 \%$ nonthermal pressure due to turbulence (Tsai et al. 1994; Evrard et al. 1996; Nagai et al. 2007). While we would not advocate the routine use of objects as morphologically disturbed as M 84 (Finoguenov \& Jones 2001) or NGC 4636 (Jones et al. 2002) for mass analysis (for some of the resulting issues see Brighenti \& Mathews 1997), taken together with our work, these results illustrate that there is little evidence, either observational or theoretical, that mild morphological disturbances in the X-ray image translate into errors on the derived mass profile that are much larger than the other systematic errors we assessed in this paper. This point speaks to the recent criticisms of Diehl \& Statler (2007), who argued that the lack of a correlation between the ellipticities of the X-ray and optical isophotes for a galaxy sample indicated the ubiquity of strong deviations from hydrostatic equilibrium. In part, the lack of such a correlation was driven by the inclusion of a significant number of objects with X-ray features carved by AGN ejecta. As discussed above, while these disturb the X-ray morphologies, they may not lead to substantial violations of hydrostatic equilibrium. Furthermore, as pointed out by Brighenti et al. (2009), highly subsonic turbulence and gas rotation (far below the level at which the enclosed mass inferred from hydrostatic techniques is significantly biased) can substantially affect the X-ray ellipticity at the small scales studied by Diehl \& Statler (2007), thus seriously undermining the premise of their study.

Notwithstanding the small amount of nonthermal pressure implied by our results, larger, albeit still modest, discrepancies have been reported between the mass inferred from stellar dynamics and hydrostatic X-ray methods at large scales in some systems (Shen \& Gebhardt 2009; Romanowsky et al. 2009). It is unclear to what extent the discrepancies arise due to deviations from hydrostatic equilibrium in the studied systems and to what extent systematic errors in the stellar modeling play a role (especially those due to triaxiality and the uncertain inclination of the galaxies). Shen \& Gebhardt argue that the stellar dynamics in one system overestimates the mass inferred from X-rays, allowing room for nonthermal pressure but only if the IMF is closer to a Salpeter than a Kroupa form. Conversely, Romanowsky et al. argue that the mass inferred from globular cluster dynamics in another system, NGC 1407, underestimates the $\mathrm{X}$-ray measurement. This is more difficult to reconcile with deviations from hydrostatic equilibrium, unless the gas is globally outflowing within the central $\sim 20 \mathrm{kpc}$ while maintaining a high X-ray luminosity and smooth-looking $\mathrm{X}$-ray isophotes (H06). Nevertheless, these studies can be used to place, entirely independently of our discussion of the SSP models, strict upper limits on the systematic errors expected for general use of the X-ray method. This would imply the bias on $M_{\mathrm{BH}}$ is no larger than \pm 0.3 dex but, as discussed above, we expect the actual systematic errors due to the hydrostatic approximation to be far smaller in the present paper. Ultimately direct observational constraints on the ubiquity of turbulent nonthermal pressure in the ISM of early-type galaxies and galaxy clusters should become possible with the advent of highresolution non-dispersive X-ray spectroscopy, as enabled by Astro- $H$ (Takahashi et al. 2008) and the International $X$-ray Observatory (IXO; White et al. 2009).

\subsection{Traditional Analysis}

In this paper, we derived constraints on the mass profiles of a sample of early-type galaxies by a "forward-fitting" approach, wherein we parameterized the mass and entropy profiles and used them to derive density and temperature profile models to fit to the data. In this section, we discuss an alternative, more traditional approach that has widely been used to model 
the mass profiles of galaxy clusters. We demonstrate that it gives consistent results with our preferred approach, albeit with larger systematic uncertainties. This method involves first fitting parameterized models to the density and temperature profiles, and then inserting these models into the equation of hydrostatic equilibrium

$$
M(<r)=r \frac{k T}{G \mu m_{H}}\left(-\frac{d \ln \rho}{d \ln r}-\frac{d \ln T}{d \ln r}\right)
$$

to infer the mass (e.g., Lewis et al. 2003). Here $G$ is the universal gravitational constant, $\mu$ is the mean molecular weight of the gas $(\sim 0.62)$, and $m_{H}$ is the mass of hydrogen.

The problem with the traditional approach is that the mass is primarily determined by the derivatives of arbitrary functions fitted to the binned density and temperature data. In principle, there exists an infinite family of parameterized models, each having an arbitrary number of maxima and minima in any given data bin, that, when averaged over the bin, equally well fit the data but which correspond to different mass profiles (many of which may be unphysical). The fine detail of the true density and temperature profiles can be disguised by the binning, and may not be produced by an ad hoc, smooth parameterized model. This problem is particularly serious when the temperature and density data points have large errors since the data may appear adequately fitted by a very smooth model that does not, for any set of its parameters, resemble the true profile for the inferred mass distribution. In such circumstances, the range of mass profiles statistically allowed may not even bracket the true mass distribution. These systematic errors (e.g., Gastaldello et al. 2007b) were the prime motivation for the forward-fitting method we introduced in $\mathrm{H} 06$ and H08 (and used in the present work), since those methods enforce a physical mass distribution. Furthermore, the models employed in the present paper only involve adopting an ad hoc parameterization for the entropy profile; hydrostatic equilibrium requires this to rise monotonically, thus precluding the erratically rising and falling shapes which can confound attempts to parameterize the binned temperature and density profiles.

Despite these concerns, the traditional method can be of use if the temperature and density profiles are sufficiently close to a smooth, parameterized form to provide a (frequentist) consistency check on our more formal (Bayesian) methods. We therefore selected appropriate, albeit arbitrary, smooth models to fit the density and temperature data (described in detail in the appendix), and hence derived mass "data points" from Equation (4), as described in Gastaldello et al. (2007b). The choice of model was not only dictated by its ability to match the temperature or density data, but also to generate a mass model which broadly resembles the expected form. The bestfitting temperature and density models are shown in Figure 3, and the derived mass "data points" are shown in Figure 7. Clearly, there is excellent agreement between the forward-fitting and traditional profiles, giving us confidence that the mass models adopted in Section 6 are not seriously in error. Notable deviations between the two methods are only seen in NGC 4261, outside $\sim 15 \mathrm{kpc}$, which likely reflects the limitations of our ad hoc density and temperature parameterizations.

To quantify the ability of the traditional method to place constraints on $M_{\mathrm{BH}}$, we fitted the set of mass "data points" shown in Figure 7 with a model comprising the dark matter halo, the stellar mass and the black hole (the gas mass is negligible over the radial span of the data; Figure 7). We obtained a good fit for all three galaxies $\left(\chi^{2} /\right.$ dof $=5.1 / 4$,
8.1/7 and 13.9/8 for NGC 1332, NGC 4261 and NGC 4472, respectively $)$, and measured $M_{\mathrm{BH}}=(1.00 \pm 0.39) \times 10^{9} M_{\odot}$, $(1.2 \pm 0.2) \times 10^{9} M_{\odot}$ and $(0.32 \pm 0.23) \times 10^{9} M_{\odot}$, respectively, while the stellar $M / L_{J}$ is $1.05 \pm 0.08 M_{\odot} L_{\odot}^{-1}, 1.57 \pm 0.03$ $M_{\odot} L_{\odot}^{-1}$ and $1.40 \pm 0.07 M_{\odot} L_{\odot}^{-1}$, respectively. Although these measurements are broadly similar to those obtained from the forward-fitting method, it is clear that the error bars do not uniformly overlap between the two approaches. This is because the small error bars obtained from the traditional method actually mask the much larger systematic uncertainties discussed above. To gain an insight into the magnitude of these effects, we cycled through each of the density and temperature parameterizations given in the Appendix, and recomputed the mass profiles, provided these models were able to fit the data adequately well. This cycling had a dramatic effect on the mass profiles, with some models even implying unphysical negative masses for some parts of the radial range. Fitting the mass models directly to the derived mass data points for each parameterization, we found that $M_{\mathrm{BH}}$ varied from its "best-fitting" value by $10^{9} M_{\odot}, 1.2 \times 10^{9} M_{\odot}$, and $0.7 \times 10^{9} M_{\odot}$, respectively, for NGC 1332, NGC 4261, and NGC 4472. Similarly, $M / L_{J}$ varied from its "best-fitting" value by $0.16 M_{\odot} L_{\odot}^{-1}, 1.6 M_{\odot} L_{\odot}^{-1}$, and $0.35 M_{\odot} L_{\odot}^{-1}$, respectively. These large changes indicate that systematic, rather than statistical, errors dominate the traditional measurements for these galaxies.

\subsection{Bondi Accretion Rates}

Given the reliability of our three-dimensional gas temperature and density models (as inferred from the accuracy of our mass fits), we were able to estimate the Bondi accretion rate for each galaxy (Di Matteo et al. 2003; Pellegrini 2005; Allen et al. 2006) without relying on the ad hoc extrapolations sometimes employed. For adiabatic, spherically symmetric accretion from infinity onto an SMBH, the accretion rate is given by Frank et al. (1992)

$$
\begin{aligned}
\dot{M}_{\text {bondi }} & =\pi G^{2} M_{\mathrm{BH}}^{2} \frac{\rho_{g}}{c_{s}^{3}}=\frac{\pi G^{2} M_{\mathrm{BH}}^{2}\left(\frac{3}{5} \mu m_{H}\right)^{\frac{3}{2}}}{\left(\rho_{g}^{-\frac{2}{3}} k T\right)^{\frac{3}{2}}} \\
& =0.0126\left(\frac{M_{\mathrm{BH}}}{10^{9} M_{\odot}}\right)^{2} s^{-\frac{3}{2}} M_{\odot} \mathrm{yr}^{-1},
\end{aligned}
$$

where $c_{s}$ is the adiabatic sound speed of the gas, and $\mathrm{s}$ is the entropy proxy (in $\mathrm{keV} \mathrm{cm}^{2}$ ), which is conserved by an adiabatic flow. If we assume that an adiabatic Bondi flow exists inward of some "transition radius," one can then compute the accretion rate from $M_{\mathrm{BH}}$ and the entropy measured at any point in the flow (and without needing to know explicitly the transition radius). Clearly, this is only a crude estimate of the accretion rate since the calculation ignores radiative losses in the flow and neglects properly accounting for the boundary conditions at the transition radius. Furthermore, our models assume that hydrostatic equilibrium holds at all radii so that if such a Bondi flow exists it could introduce systematic errors into our calculation of $M_{\mathrm{BH}}$ from the X-rays. Nevertheless, as discussed in Section 8.1, such errors do not appear large.

Allen et al. (2006) assumed that the transition to a Bondi flow occurs at the "accretion radius," $r_{A}\left(=2 G M_{\mathrm{BH}} / c_{s}^{2}(\infty)\right.$, where $c_{s}(\infty)$ is the sound speed far from the region of influence of the SMBH; Frank et al. 1992), that is, the point at which the gas flow becomes supersonic in a conventional Bondi flow. For only one of our galaxies, we can actually resolve $r_{A}$ (for NGC 4649 
Table 4

Bondi Accretion Rates

\begin{tabular}{ccc}
\hline \hline Galaxy & $\begin{array}{c}r_{A} \\
(\mathrm{pc})\end{array}$ & $\begin{array}{c}\log _{10} \dot{M}_{\text {bondi }} \\
\left(\left[M_{\odot} \mathrm{yr}^{-1}\right]\right)\end{array}$ \\
\hline NGC 1332 & $45_{-25}^{+36}$ & $-1.94_{-0.76}^{+0.66}$ \\
NGC 4261 & $24_{-13}^{+15}$ & $-2.26_{-0.57}^{+0.56}$ \\
NGC 4472 & $33_{-17}^{+31}$ & $-2.3_{-0.65}^{+0.66}$ \\
NGC 4649 & $165_{-54}^{+33}$ & $-1.41_{-0.33}^{+0.29}$ \\
\hline
\end{tabular}

Notes. Accretion radii and Bondi accretion rates for each galaxy, computed from our fitted density and temperature models (see the text). Error bars correspond to $90 \%$ confidence limits.

it is $\sim 160 \mathrm{pc} \simeq 2^{\prime \prime}$ ) and the entropy profile is, indeed, very close to adiabatic at this scale (H08). Still, the best-fitting $s_{0}$ for the other galaxies is nonzero, suggesting a similar flattening of the entropy profiles at the smallest (unresolved) scales. We therefore found it convenient to use the asymptotic $(r \rightarrow 0)$ value of $s$ to evaluate Equation (5). We list the most probable, marginalized values and $90 \%$ confidence regions for $\dot{M}_{\text {bondi }}$ in Table 4. Allen et al. (2006) also evaluated $\dot{M}_{\text {bondi }}$ for NGC 4472 and their reported value agrees, within errors, with our result.

For a sample of galaxies, Allen et al. (2006) found that $\dot{M}_{\text {bondi }}$ correlates tightly with an estimate of the total AGN power ejected in the jets. This jet power was estimated under the assumption that the thermal energy of the ISM is increased by the work done in creating cavities with a relativistic gas, so that the mean power in the jet can be obtained from the gas pressure, the cavity size and an estimate of the time to create it. Mathews \& Brighenti (2008) argued, however, that cavities created with cosmic rays do not heat the gas in this way and should, instead drive large-scale circulation of the gas. In this case, the jet power calculation is likely a very inaccurate way to estimate the energy injected into the ISM by the AGN. Despite these concerns, the tight correlation found by Allen et al. (2006) is intriguing, and it is interesting to investigate whether the galaxies in our sample are consistent with it.

The values of $M_{\text {bondi }}$ in Table 4 are comparable with the medium-to-high accretion rate systems reported by Allen et al. (2006), implying that the jet power should be considerable for all of these systems if they lie on the same relation. While there is clear evidence of AGN-blown cavities in NGC 4261 and NGC 4472, the situation is much less clear for the other two systems, which have higher $\dot{M}_{\text {bondi }}$. The X-ray image of NGC 1332 is smooth and relaxed and exhibits little or no evidence of X-ray cavities (Figure 1). Nonetheless, the modest X-ray surface brightness of this object, coupled with the lack of obvious radio lobes (given the weak emission in the NVSS image) makes the identification of small, low-contrast $\mathrm{X}$-ray depressions challenging. More significant, however, is the absence of cavities in a deep Chandra observation of the X-ray bright NGC 4649 (H08). Based on much shallower data, Shurkin et al. (2008) claimed to find small X-ray cavities (which we did not confirm), but even taking these at face value, the implied jet power estimate is only $\sim 1.3 \times 10^{42} \mathrm{erg} \mathrm{s}^{-1}$. For this system, therefore, the jet power is, at most, comparable to the lowest values found by Allen et al. (2006), while $\dot{M}_{\text {bondi }}$ compares to their highest Bondi rates, in stark discrepancy with their correlation. The existence of galaxies with high $\dot{M}_{\text {bondi }}$ but no cavities suggests that the Allen et al. correlation takes time to be established; we may be observing NGC 4649 and NGC 1332 at an unusual time, while the AGN is just turning on and the radio lobes are beginning to inflate.

\section{CONCLUSIONS}

We have presented new hydrostatic, X-ray models for the centers of three early-type galaxies observed with Chandra. Combined with our recent study of the elliptical galaxy NGC 4649 (H08), we found the following, in summary.

1. The black hole masses measured by our method are in agreement with those obtained by stellar or gas dynamics techniques. This provides support not only for our new $\mathrm{X}$-ray approach, but also for the accuracy of the dynamical methods.

2. Like in NGC 4649, the black hole in NGC 4472 is unambiguously required by our mass models; even with a conservative, flat prior for $M_{\mathrm{BH}}$, it is detected at the $\sim 2.3 \sigma$ level.

3. Accurate stellar stellar $M / L_{J}$ ratios require careful modeling of the stellar mass component; with our detailed models, we obtained stellar $M / L_{J}$ ratios in agreement with the predictions of single-burst stellar population synthesis (SSP) models computed for a Kroupa or Chabrier IMF. This leaves little room for the steepening of the central dark matter density profile predicted by models of "adiabatic contraction," unless it exists in a conspiracy with nonthermal pressure.

4. Taking the SSP models at the face value, this agreement suggests the gas is very close to hydrostatic, and nonthermal pressure can provide no more than $\sim 10 \%-20 \%$ of the total support, unless there is a conspiracy between the shape of the IMF and nonthermal pressure.

5. The two galaxies with the highest Bondi accretion rates exhibit little or no evidence of X-ray cavities, suggesting that the Allen et al. (2006) correlation with the AGN jet power takes time to be established.

We would like to thank Greg Martinez, Aaron Barth, Hélène Flohic, and Sebastian Heinz for helpful discussions. This research has made use of data obtained from the High Energy Astrophysics Science Archive Research Center (HEASARC), provided by NASA's Goddard Space Flight Center. Some of the data presented in this paper were obtained from the Multimission Archive at the Space Telescope Science Institute (MAST). STScI is operated by the Association of Universities for Research in Astronomy, Inc., under NASA contract NAS5-26555. This research has also made use of the NASA/IPAC Extragalactic Database (NED) which is operated by the Jet Propulsion Laboratory, California Institute of Technology, under contract with NASA, and the HyperLEDA database (http://leda.univlyon1.fr). Partial support for this work was provided by NASA under grant NNG04GE76G issued through the Office of Space Sciences Long-Term Space Astrophysics Program. Partial support was also provided by NASA through Chandra Award number G07-8083X issued by the Chandra X-Ray Center, which is operated by the Smithsonian Astrophysical Observatory for and on behalf of NASA.

\section{APPENDIX}

\section{TRADITIONAL ANALYSIS MODELS}

We here outline the explicit models used to parameterize the density and temperature profiles in our "traditional analysis" (Section 8.4). These models are entirely ad hoc and were motivated only insofar as they reasonably capture the global shape of the appropriate profile. In practice, the particular combination of density and temperature model preferred to fit 
Table 5

Traditional Analysis Models

\begin{tabular}{|c|c|c|c|c|c|c|c|}
\hline Galaxy & Model & $\begin{array}{c}A_{1} \\
\left(\mathrm{~g} \mathrm{~cm}^{-3} ; \mathrm{keV}\right) \\
\end{array}$ & $\begin{array}{c}A_{2} \\
\left(\mathrm{~g} \mathrm{~cm}^{-3} ; \mathrm{keV}\right) \\
\end{array}$ & $\begin{array}{c}R_{c} \\
(\mathrm{kpc})\end{array}$ & $\begin{array}{c}R_{c 2} \\
(\mathrm{kpc})\end{array}$ & $\alpha$ & $\beta$ \\
\hline \multicolumn{8}{|c|}{ Density Models } \\
\hline NGC 1332 & Equation (A1) & $(4.6 \pm 0.7) \times 10^{-25}$ & $\ldots$ & 0.25 & $\ldots$ & $0.98 \pm 0.07$ & $0.47 \pm 0.008$ \\
\hline NGC 4261 & Equation (A2) & $(1.6 \pm 0.1) \times 10^{-24}$ & $(2.1 \pm 0.4) \times 10^{-27}$ & $0.198 \pm 0.008$ & $42 \pm 8$ & $\ldots$ & $0.543 \pm 0.007$ \\
\hline NGC 4472 & Equation (A2) & $(7.9 \pm 1) \times 10^{-25}$ & $(6.4 \pm 3.4) \times 10^{-27}$ & $0.25 \pm 0.02$ & $15 \pm 18$ & $\ldots$ & $0.48 \pm 0.02$ \\
\hline \multicolumn{8}{|c|}{ kT Models } \\
\hline NGC 1332 & Equation (A3) & $0.39 \pm 0.02$ & $0.3 \pm 0.06$ & $2.1 \pm 1.4$ & $\ldots$ & $5 \pm 0.5$ & \\
\hline NGC 4261 & Equation (A4) & $0.609 \pm 0.006$ & $0.75 \pm 0.06$ & $6.4 \pm 0.6$ & $\ldots$ & $2.8 \pm 0.4$ & \\
\hline NGC 4472 & Equation (A4) & $0.65 \pm 0.01$ & $0.42 \pm 0.05$ & $4 \pm 0.5$ & $\ldots$ & $2 \pm 1$ & \\
\hline
\end{tabular}

Notes. Best-fitting results for the arbitrary, parameterized models to the temperature and density profiles used in our "traditional" analysis (Section 8.4). For each galaxy, we identify which of the functional forms given in the Appendix are used ("Model") and, for each model parameter, the best-fitting values and $1 \sigma$ errors.

the Chandra data of each galaxy (which is given in Table 5) was chosen as that which most accurately reproduces the mass profile derived in Section 6.

To fit the density profile, we used either a "cusped beta model,"

$$
\rho=A_{1}\left(\frac{R}{R_{c}}\right)^{-\alpha}\left(1+\frac{R^{2}}{R_{c}^{2}}\right)^{-\frac{3}{2} \beta+\frac{1}{2} \alpha}
$$

or a "double beta model,"

$$
\rho=\sqrt{A_{1}^{2}\left(1+\frac{R^{2}}{R_{c}^{2}}\right)^{-3 \beta}+A_{2}^{2}\left(1+\frac{R^{2}}{R_{c 2}^{2}}\right)^{-3 \beta}}
$$

and to fit the temperature data we adopted one of the following arbitrary models:

$$
\begin{gathered}
k T=A_{1}+A_{2}\left(1+\frac{R}{R_{c}}\right)^{-\alpha} \\
k T=A_{1}+A_{2}\left(1+\left[\frac{R}{R_{c}}\right]^{-\alpha}\right)^{-1} \text { (A3) } \\
k T=A_{1}\left(\frac{R}{R_{c}}\right)^{\alpha} \exp \left(-\frac{R}{R_{c}}\right)+A_{2}\left(\frac{R}{R_{c}}\right)^{\beta}\left(1-\exp \left[-\frac{R}{R_{c}}\right]\right)
\end{gathered}
$$

The best-fitting parameters for each galaxy are given in Table 5 .

\section{REFERENCES}

Abadi, M. G., Navarro, J. F., Fardal, M., Babul, A., \& Steinmetz, M. 2009, arXiv:0902.2477

Allen, S. W. 1998, MNRAS, 296, 392

Allen, S. W., Dunn, R. J. H., Fabian, A. C., Taylor, G. B., \& Reynolds, C. S. 2006, MNRAS, 372, 21

Anders, E., \& Grevesse, N. 1989, Geochim. Cosmochim. Acta, 53, 197

Arnaud, K. A. 1996, in ASP Conf. Ser. 101, Astronomical Data Analysis Software and Systems V, ed. G. H. Jacoby \& J. Barnes (San Francisco, CA: ASP), 17

Asplund, M., Grevesse, N., \& Sauval, J. 2005, in ASP Conf. Ser. 336, Cosmic Abundances as Records of Stellar Evolution and Nucleosynthesis, ed. T. G. Barnes \& F. N. Bash (San Francisco, CA: ASP), 25

Becker, R. H., White, R. L., \& Helfand, D. J. 1995, ApJ, 450, 559

Bender, R., Saglia, R. P., \& Gerhard, O. E. 1994, MNRAS, 269, 785

Biller, B. A., Jones, C., Forman, W. R., Kraft, R., \& Ensslin, T. 2004, ApJ, 613, 238

Binney, J. J., Davies, R. L., \& Illingworth, G. D. 1990, ApJ, 361, 78

Blumenthal, G. R., Faber, S. M., Flores, R., \& Primack, J. R. 1986, ApJ, 301, 27

Brighenti, F., \& Mathews, W. G. 1997, ApJ, 486, L83
Brighenti, F., \& Mathews, W. G. 1999, ApJ, 527, L89

Brighenti, F., Mathews, W. G., Humphrey, P. J., \& Buote, D. A. 2009, ApJ, submitted

Bruzual, G., \& Charlot, S. 2003, MNRAS, 344, 1000

Buote, D. A. 2002, ApJ, 574, L135

Buote, D. A., \& Canizares, C. R. 1996, ApJ, 457, 177

Buote, D. A., Gastaldello, F., Humphrey, P. J., Zappacosta, L., Bullock, J. S., Brighenti, F., \& Mathews, W. G. 2007, ApJ, 664, 123

Caon, N., Macchetto, D., \& Pastoriza, M. 2000, ApJS, 127, 39

Cappellari, M., Neumayer, N., Reunanen, J., van der Werf, P. P., de Zeeuw, P. T., \& Rix, H.-W. 2009, MNRAS, 394, 660

Cappellari, M., Verolme, E. K., van der Marel, R. P., Kleijn, G. A. V., Illingworth, G. D., Franx, M., Carollo, C. M., \& de Zeeuw, P. T. 2002, ApJ, 578, 787

Cash, W. 1979, ApJ, 228, 939

Chabrier, G. 2003, PASP, 115, 763

Churazov, E., Forman, W., Vikhlinin, A., Tremaine, S., Gerhard, O., \& Jones, C. 2008, MNRAS, 388, 1062

Condon, J. J., Cotton, W. D., Greisen, E. W., Yin, Q. F., Perley, R. A., Taylor, G. B., \& Broderick, J. J. 1998, AJ, 115, 1693

Conroy, C., \& Wechsler, R. H. 2009, ApJ, 696, 620

Croston, J. H., Hardcastle, M. J., \& Birkinshaw, M. 2005, MNRAS, 357, 279

Di Matteo, T., Allen, S. W., Fabian, A. C., Wilson, A. S., \& Young, A. J. 2003, ApJ, 582, 133

Di Matteo, T., Springel, V., \& Hernquist, L. 2005, Nature, 433, 604

Dickey, J. M., \& Lockman, F. J. 1990, ARA\&A, 28, 215

Diehl, S., \& Statler, T. S. 2007, ApJ, 668, 150

Dressler, A., \& Sandage, A. 1983, ApJ, 265, 664

Dutton, A. A., van den Bosch, F. C., Dekel, A., \& Courteau, S. 2007, ApJ, 654, 27

Evrard, A. E., Metzler, C. A., \& Navarro, J. F. 1996, ApJ, 469, 494

Fang, T., Humphrey, P., \& Buote, D. 2009, ApJ, 691, 1648

Feroz, F., \& Hobson, M. P. 2008, MNRAS, 384, 449

Feroz, F., Hobson, M. P., \& Bridges, M. 2008, arXiv:0809.3437

Ferrarese, L., \& Ford, H. 2005, Space Sci. Rev., 116, 523

Ferrarese, L., Ford, H. C., \& Jaffe, W. 1996, ApJ, 470, 444

Ferrarese, L., \& Merritt, D. 2000, ApJ, 539, L9

Finoguenov, A., \& Jones, C. 2001, ApJ, 547, L107

Finoguenov, A., Ponman, T. J., Osmond, J. P. F., \& Zimer, M. 2007, MNRAS, 374,737

Fioc, M., \& Rocca-Volmerange, B. 1997, A\&A, 326, 950

Floyd, D. J. E., et al. 2008, ApJS, 177, 148

Frank, J., King, A., \& Raine, D. 1992, Accretion Power in Astrophysics (2nd ed.; Cambridge: Cambridge Univ. Press)

Gastaldello, F., Buote, D. A., Humphrey, P. J., Zappacosta, L., Brighenti, F., \& Mathews, W. G. 2007a, in Heating Versus Cooling in Galaxies and Clusters of Galaxies, ed. H. Böhringer et al. (Berlin: Springer), 275

Gastaldello, F., Buote, D. A., Humphrey, P. J., Zappacosta, L., Bullock, J. S., Brighenti, F., \& Mathews, W. G. 2007b, ApJ, 669, 158

Gavazzi, R. 2005, A\&A, 443, 793

Gebhardt, K., \& Thomas, J. 2009, ApJ, 700, 1690

Gebhardt, K., et al. 1996, AJ, 112, 105

Gebhardt, K., et al. 2000a, ApJ, 539, L13

Gebhardt, K., et al. 2000b, AJ, 119, 1157

Gebhardt, K., et al. 2003, ApJ, 583, 92

Gnedin, O. Y., Kravtsov, A. V., Klypin, A. A., \& Nagai, D. 2004, ApJ, 616, 16 
Gnedin, O. Y., Weinberg, D. H., Pizagno, J., Prada, F., \& Rix, H.-W. 2007, ApJ, 671,1115

Graham, A. W. 2008, Publ. Astron. Soc. Aust., 25, 167

Graham, A. W., Erwin, P., Caon, N., \& Trujillo, I. 2001, ApJ, 563, L11

Granato, G. L., De Zotti, G., Silva, L., Bressan, A., \& Danese, L. 2004, ApJ, 600,580

Gültekin, K., et al. 2009, ApJ, 698, 198

Hernquist, L. 1990, ApJ, 356, 359

Hopkins, P. F., Hernquist, L., Cox, T. J., Di Matteo, T., Robertson, B., \& Springel, V. 2006, ApJS, 163, 1

Hu, J. 2008, MNRAS, 386, 2242

Humphrey, P. J. 2009, ApJ, 690, 512

Humphrey, P. J., \& Buote, D. A. 2006, ApJ, 639, 136

Humphrey, P. J., \& Buote, D. A. 2008, ApJ, 689, 983

Humphrey, P. J., Buote, D. A., Brighenti, F., Gebhardt, K., \& Mathews, W. G. 2008, ApJ, 683, 161 (H08)

Humphrey, P. J., Buote, D. A., \& Canizares, C. R. 2004, ApJ, 617, 1047

Humphrey, P. J., Buote, D. A., Gastaldello, F., Zappacosta, L., Bullock, J. S., Brighenti, F., \& Mathews, W. G. 2006, ApJ, 646, 899 (H06)

Humphrey, P. J., Liu, W., \& Buote, D. A. 2009, ApJ, 693, 822

Irwin, J. A., Athey, A. E., \& Bregman, J. N. 2003, ApJ, 587, 356

Irwin, J. A., \& Sarazin, C. L. 1996, ApJ, 471, 683

Jensen, J. B., Tonry, J. L., Barris, B. J., Thompson, R. I., Liu, M. C., Rieke, M. J., Ajhar, E. A., \& Blakeslee, J. P. 2003, ApJ, 583, 712

Jetha, N. N., Ponman, T. J., Hardcastle, M. J., \& Croston, J. H. 2007, MNRAS, 376, 193

Jones, C., Forman, W., Vikhlinin, A., Markevitch, M., David, L., Warmflash, A., Murray, S., \& Nulsen, P. E. J. 2002, ApJ, 567, L115

King, A. 2003, ApJ, 596, L27

Kormendy, J., Fisher, D. B., Cornell, M. E., \& Bender, R. 2009, ApJS, 182, 216

Kormendy, J., \& Richstone, D. 1995, ARA\&A, 33, 581

Krajnović, D., Cappellari, M., Emsellem, E., McDermid, R. M., \& de Zeeuw, P. T. 2005, MNRAS, 357, 1113

Kronawitter, A., Saglia, R. P., Gerhard, O., \& Bender, R. 2000, A\&AS, 144, 53

Kroupa, P. 2001, MNRAS, 322, 231

Lauer, T. R., et al. 2007, ApJ, 662, 808

Lewis, A. D., Buote, D. A., \& Stocke, J. T. 2003, ApJ, 586, 135

Lucy, L. B. 1974, AJ, 79, 745

Macciò, A. V., Dutton, A. A., \& van den Bosch, F. C. 2008, MNRAS, 391, 1940

Mahdavi, A., Hoekstra, H., Babul, A., \& Henry, J. P. 2008, MNRAS, 384, 1567

Maraston, C. 2005, MNRAS, 362, 799

Marconi, A., \& Hunt, L. K. 2003, ApJ, 589, L21

Marconi, A., Pastorini, G., Pacini, F., Axon, D. J., Capetti, A., Macchetto, D., Koekemoer, A. M., \& Schreier, E. J. 2006, A\&A, 448, 921

Markowitz, A., et al. 2003, ApJ, 593, 96

Mathews, W. G., \& Brighenti, F. 2003, ARA\&A, 41, 191

Mathews, W. G., \& Brighenti, F. 2008, ApJ, 685, 128

Merritt, D., \& Ferrarese, L. 2001, ApJ, 547, 140

Miyoshi, M., Moran, J., Herrnstein, J., Greenhill, L., Nakai, N., Diamond, P., \& Inoue, M. 1995, Nature, 373, 127

Nagai, D., Vikhlinin, A., \& Kravtsov, A. V. 2007, ApJ, 655, 98

Navarro, J. F., Frenk, C. S., \& White, S. D. M. 1997, ApJ, 490, 493

Neumayer, N., Cappellari, M., Reunanen, J., Rix, H.-W., van der Werf, P. P., de Zeeuw, P. T., \& Davies, R. I. 2007, ApJ, 671, 1329

Nipoti, C., Londrillo, P., \& Ciotti, L. 2003, MNRAS, 342, 501

Noel-Storr, J., Baum, S. A., Verdoes Kleijn, G., van der Marel, R. P., O'Dea, C. P., de Zeeuw, P. T., \& Carollo, C. M. 2003, ApJS, 148, 419

O’Neill, P. M., Nandra, K., Papadakis, I. E., \& Turner, T. J. 2005, MNRAS, 358, 1405
Peletier, R. F., Davies, R. L., Illingworth, G. D., Davis, L. E., \& Cawson, M. 1990, AJ, 100, 1091

Pellegrini, S. 2005, ApJ, 624, 155

Piffaretti, R., Jetzer, P., \& Schindler, S. 2003, A\&A, 398, 41

Poulain, P. 1988, A\&AS, 72, 215

Poulain, P., \& Nieto, J.-L. 1994, A\&AS, 103, 573

Press, W. H., Teukolsky, S. A., Vetterling, W. T., \& Flannery, B. P. 1992, Numerical Recipes in C: The Art of Scientific Computing (2nd ed.; Cambridge: Cambridge Univ. Press)

Prugniel, P., \& Simien, F. 1997, A\&A, 321, 111

Rembold, S. B., Pastoriza, M. G., \& Bruzual, G. 2005, A\&A, 436, 57

Revnivtsev, M., Churazov, E., Sazonov, S., Forman, W., \& Jones, C. 2008, A\&A, 490, 37

Robertson, B., Hernquist, L., Cox, T. J., Di Matteo, T., Hopkins, P. F., Martini, P., \& Springel, V. 2006, ApJ, 641, 90

Romanowsky, A. J., Strader, J., Spitler, L. R., Johnson, R., Brodie, J. P., Forbes, D. A., \& Ponman, T. 2009, AJ, 137, 4956

Sánchez-Blázquez, P., Forbes, D. A., Strader, J., Brodie, J., \& Proctor, R. 2007, MNRAS, 377, 759

Sandage, A., \& Visvanathan, N. 1978, ApJ, 223, 707

Scharf, C. A., Zurek, D. R., \& Bureau, M. 2005, ApJ, 633, 154

Shapiro, K. L., Cappellari, M., de Zeeuw, T., McDermid, R. M., Gebhardt, K., van den Bosch, R. C. E., \& Statler, T. S. 2006, MNRAS, 370, 559

Shen, J., \& Gebhardt, K. 2009, submitted

Shurkin, K., Dunn, R. J. H., Gentile, G., Taylor, G. B., \& Allen, S. W. 2008, MNRAS, 383, 923

Silge, J. D., Gebhardt, K., Bergmann, M., \& Richstone, D. 2005, AJ, 130, 406

Silk, J., \& Rees, M. J. 1998, A\&A, 331, L1

Siopis, C., et al. 2009, ApJ, 693, 946

Skilling, J. 2004, in AIP Conf. Proc. 735, Bayesian Inference and Maximum Entropy Methods in Science and Engineering, ed. R. Fischer, R. Preuss, \& U. von Toussaint (Melville, NY: AIP), 395

Sun, M., Voit, G. M., Donahue, M., Jones, C., \& Forman, W. 2009, ApJ, 693, 1142

Takahashi, T., et al. 2008, Proc. SPIE, 7011, 701100

Thomas, J., Saglia, R. P., Bender, R., Thomas, D., Gebhardt, K., Magorrian, J., Corsini, E. M., \& Wegner, G. 2007, MNRAS, 382, 657

Tonry, J. L., Dressler, A., Blakeslee, J. P., Ajhar, E. A., Fletcher, A., Luppino, G. A., Metzger, M. R., \& Moore, C. B. 2001, ApJ, 546, 681

Trager, S. C., Faber, S. M., Worthey, G., \& González, J. J. 2000, AJ, 119, 1645

Tremaine, S., et al. 2002, ApJ, 574, 740

Tsai, J. C., Katz, N., \& Bertschinger, E. 1994, ApJ, 423, 553

Valluri, M., Merritt, D., \& Emsellem, E. 2004, ApJ, 602, 66

van den Bosch, R. C. E., van de Ven, G., Verolme, E. K., Cappellari, M., \& de Zeeuw, P. T. 2008, MNRAS, 385, 647

van den Bosch, R. C. E., et al. 2009, MNRAS, submitted

van der Marel, R. P., Cretton, N., de Zeeuw, P. T., \& Rix, H.-W. 1998, ApJ, 493, 613

van der Marel, R. P., \& Franx, M. 1993, ApJ, 407, 525

Verdoes Kleijn, G. A., van der Marel, R. P., de Zeeuw, P. T., Noel-Storr, J., \& Baum, S. A. 2002, AJ, 124, 2524

Vikhlinin, A., Kravtsov, A., Forman, W., Jones, C., Markevitch, M., Murray, S. S., \& Van Speybroeck, L. 2006, ApJ, 640, 691

White, N. E., et al. 2009, AAS Meeting Abstracts, 213, 457.01

Windhorst, R. A., et al. 2006, New Astron. Rev., 50, 121

Wyithe, J. S. B. 2006, MNRAS, 365, 1082

Zappacosta, L., Buote, D. A., Gastaldello, F., Humphrey, P. J., Bullock, J., Brighenti, F., \& Mathews, W. 2006, ApJ, 650, 777

Zezas, A., Birkinshaw, M., Worrall, D. M., Peters, A., \& Fabbiano, G. 2005, ApJ, 627,711 